\title{
Manifold-Based Surfaces with Boundaries
}

\author{
Elif Tosun, Denis Zorin* \\ New York University \\ 719 Broadway, 12th Fl \\ New York, NY 10003
}

\begin{abstract}
We present a manifold-based surface construction extending the $C^{\infty}$ construction of Ying and Zorin (2004a). Our surfaces allow for pircewise-smooth boundaries, have user-controlled arbitrary degree of smoothness and improved derivative and visual behavior. 2-flexibility of our surface construction is confirmed numerically for a range of local mesh configurations.
\end{abstract}

Key words: Geometric modeling, manifolds, high-order surfaces

\section{Introduction}

Manifold-based parametric surface representations, unlike most commonly used arbitrary-topology constructions (free-form splines and subdivision surfaces) are based on blending overlapping local surface approximations, rather than stitching nonoverlapping patches together. Manifold-based representations of surfaces offer a unique combination of features: arbitrary topology, arbitrary-order smoothness, flexibility, explicit analytic expressions for local parametrizations and linear dependence on control points.

High-order smoothness, while having relatively little impact on visual surface quality, is useful for numerical purposes: for example, for $C^{3}$-continuous surfaces variation of curvature functionals are well-defined everywhere and $C^{k}$-continuity makes it possible to use quadratures of order $k+1$ to ensure rapid convergence. Smooth explicit analytic parametrization makes it possible to evaluate the surface and its derivatives at arbitrary points in a straightforward manner. Flexibility, 2-flexibility in particular, is essential for good local approximation of smooth shapes.

Manifold surfaces are a natural setting for solving equations on surfaces as the chart overlap simplifies maintaining smoothness of solutions across boundaries. It is often easier to apply algorithms designed for parametric patches which treat the surface as a function $f(u, v)$ on a planar domain to surfaces of arbitrary topology without handling special cases for inter-patch boundaries needed for patch-based representations.

A number of manifold surface constructions were proposed in the past; in this paper, we present a generalization of the $C^{\infty}$ construction of Ying and Zorin (2004a), and explore the properties of resulting surfaces. Our goal is to construct surfaces with improved mathematical properties (higher derivative continuity, closed-form parametrization, flexibility) while maintaining, to the extent possible, visual appearance of Catmull-Clark surfaces.

In comparison to Ying and Zorin (2004a), our surfaces need not be closed. We define special charts and local geometry construction needed to represent surfaces with piecewise smooth boundaries, with boundary curves defined only using boundary control points.

We relax the requirement that surfaces are $C^{\infty}$ : it turns out, that by considering surfaces of arbitrarily high but finite degree of continuity, it is possible to decrease derivative magnitudes and improve surface quality. We describe geometry functions and several possible choices of blending functions for $C^{d}$-continuous surfaces.

\footnotetext{
* Corresponding author

Email addresses: elif@cs.nyu.edu, dzorin@cs.nyu.edu (Elif Tosun, Denis Zorin)
} 
One of our motivations for considering manifold-based surfaces comes from solving boundary integral equations on surfaces with high-order accuracy. Such equations arise in a variety of contexts, including electrostatic computations, electromagnetic scattering, Stokes equations and other. To achieve high-order convergence rates, a high-order smooth nonsingular parametrization is necessary. Our manifold-based surfaces are an essential part of the boundary integral equation solver described in (Ying et al., 2006), which achieves high convergence rates in part thanks to high degree of continuity of manifold-based surfaces.

This paper is organized as follows: in Section 2, we discuss related work and briefly compare our construction with other manifold constructions. Section 3 reviews the definitions of of smooth surfaces with piecewise-smooth boundaries. In Section 4 , we review the $C^{\infty}$ manifold surface construction and extend it to surfaces with piecewise-smooth boundaries. Section 5 describes local geometry functions for $C^{d}$-continuous surfaces with improved derivative behavior, with blending partition-of-unity functions discussed Section 6 . In Section 7 we examine derivative behavior, present numerical evidence of flexibility and compare visual quality of different versions of the manifold construction and subdivision surfaces.

\section{Previous Work}

A considerable number of $C^{d}, d>1$ surface constructions for arbitrary meshes of various types were proposed over years, with the important case of $d=2$ receiving most attention. Known constructions can be partitioned into three groups, based on spline patches, subdivision surfaces and manifolds respectively. We briefly review other high-order smoothness constructions and consider manifold-based constructions in more detail.

In (Hagen and Pottmann, 1989) $C^{2}$ interpolants of boundary position, tangent and curvature data are constructed. Gregory and Hahn (1989) describe a $C^{2}$ hole-filling algorithm; Bohl and Reif (1997) describe $C^{2}$ conditions on degenerate patches and how $N$ patches can be joined at a point. $C^{2}$ spline surfaces on arbitrary meshes were constructed by Peters (1996) and $C^{d}$ spline surfaces for general $d$ are described by Prautzsch (1997). More recently, various types of constructions based on polynomial patches were proposed in (Peters, 2002), (Loop, 2004) and (Karčiauskas and Peters, 2005). $C^{2}$ subdivision algorithms based on standard schemes and with zero curvature at extraordinary vertices were proposed by Prautzsch and Umlauf (1998) and Biermann et al. (2000a). Flexible $C^{2}$ modifications of subdivision surfaces are described in (Zorin, 2006a) and (Levin, 2006).

The idea of manifold-based constructions was introduced in (Grimm and Hughes, 1995) where a manifold surface is built from a polygonal mesh using one chart per mesh element (vertices, edges and faces). In their construction, all interior vertices are required to have valence four (which can be achieved by a subdivisionbased pre-process), and faces have to be polygons with no more than six edges. In (Grimm, 2002) and (Grimm and Hughes, 2003) this manifold-based construction is used to generate parametrizations, where manifolds are used as parameter domains for surfaces of different topologies.

In Navau and Garcia (2000) another manifold-based method for generating $C^{d}$ surfaces is described. This method requires a pre-process of repeated Catmull-Clark subdivision steps in order to isolate irregularities of the mesh. The number of such steps is $O(\log n)$ in worst case, where $n$ is an input parameter describing the extent of the influence of control points. Parts of regular and irregular subgraphs of the resulting mesh are chosen as charts with corresponding transition functions. The shapes and sizes of charts are dependent on the input parameter $n$ and the regularity. In order to generalize the B-spline approach with this method, each chart is then mapped to a control point which describes the shape of the resulting surface. In (Navau et al., 2002), three realizations of this general scheme is described.

Extensions of these methods for surfaces with smooth boundaries have been outlined in most constructions mentioned above. In (Grimm and Hughes, 1995) the extension involves the definition of a different set of charts for boundary edges and vertices similar to our construction. On the other hand in Navau and Garcia (2000), the extension for boundaries is achieved by adding auxiliary layers of faces around the mesh boundary such that all vertices can be treated the same. Compared to previously considered boundary constructions, our construction has the following distinctive features: boundary curves can be independent of the interior, and include corner points.

An important recent direction of work is manifold splines (Gu et al., 2005, 2006, 2007; Wang et al., 2008). Compared to other manifold constructions, manifold splines use an atlas of affine transition maps. The advantages in this case are considerable: transition maps are greatly simplified, and it becomes feasible not 


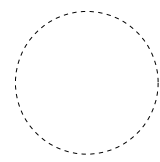

$D$

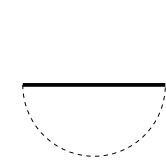

$H$

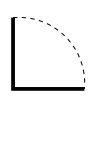

$Q_{1}$

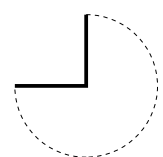

$Q_{3}$

Figure 1: The charts for a surface with piecewise smooth boundary.

to use a partition of unity to merge local geometries together: one can use a spline construction on arbitrary triangulations (e.g. DMS splines or Powell-Sabin splines) to build a purely polynomial surface; alternatively, one can fit splines defined on a regular grid in the plane. For meshes with boundary, in principle, one can construct a purely affine atlas, and for closed meshes one can construct an affine atlas after a number of points determined by the genus of the mesh are removed. One can observe that manifold splines reduces the task of building a surface of arbitrary topology to mapping the mesh to a plane and local constructions on arbitrary triangulations. While methods for these constructions are available, they are relatively complex, especially for high order smoothness. They are not commonly used for $a b$ initio construction of surfaces of high-order smoothness from relatively coarse control meshes, which is our primary goal. One can regard manifold splines and our construction as complementary, each targeting a different scope of applications.

\section{Surfaces with Boundaries}

Our first goal is to describe the manifold-based construction of surfaces with boundaries. A more general class of piecewise smooth surfaces can be easily obtained from this class by stitching smooth surfaces with boundaries along boundary curves. To be able to do this, the boundary curves have to be defined independently of the interior, i.e. the shape of the boundary curve should be completely determined by the control points on the boundary of the control mesh (Figure 3, left). We describe ways to obtain both interior-dependent and interior-independent boundaries.

Defining surfaces with boundaries requires additional chart types. Recall that for a closed $C^{1}$-continuous surface in $\mathbf{R}^{3}$, each point has a neighborhood that can be smoothly deformed (that is, using a $C^{1}$ map of maximal rank) into an open planar disk $D$. A surface with a smooth boundary can be described in a similar way, but neighborhoods of boundary points can be smoothly deformed into a half-disk $H$, with a closed boundary segment (Figure 1). In order to allow piecewise smooth boundaries, we introduce two additional types of local charts: concave and convex corner charts, $Q_{3}$ and $Q_{1}$. A $C^{1}$-continuous surface with piecewise smooth boundary looks locally like one of the domains $D, H, Q_{1}$, or $Q_{3}$. Convex and concave corners, while being equivalent topologically, are not $C^{1}$-diffeomorphic, that is, there is no $C^{1}$ non-singular map from $Q_{1}$ to $Q_{3}$. One can show that almost all $C^{1}$-continuous surfaces obtained as linear combinations of basis functions are diffeomorphic. This implies that separate constructions are needed for convex and concave boundary corners, just as it is the case for subdivision surfaces (Biermann et al., 2000a). Rather than having one type of star-shaped chart domains per valence, as it was the case for closed surfaces, we need to consider three chart types as shown in the Figure 2.

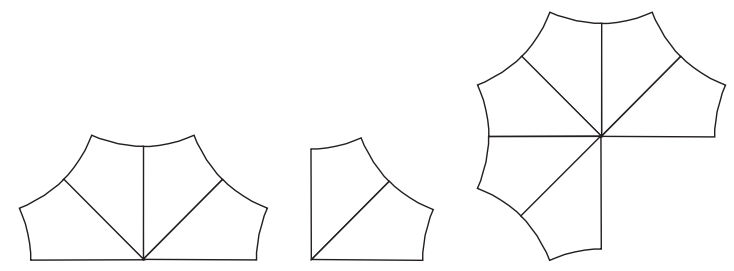

Figure 2: Left: valance 4 smooth boundary chart. Center: valance 2 convex corner chart. Right: valance 6 concave corner chart.

While the number of chart types one needs to consider increases, the main elements of the construction 
remain similar: we can use similarly constructed chart maps, partition of unity functions, and geometry functions. While geometry functions can be obtained using essentially the same fitting idea, interior-independent boundary curves require additional constraints.
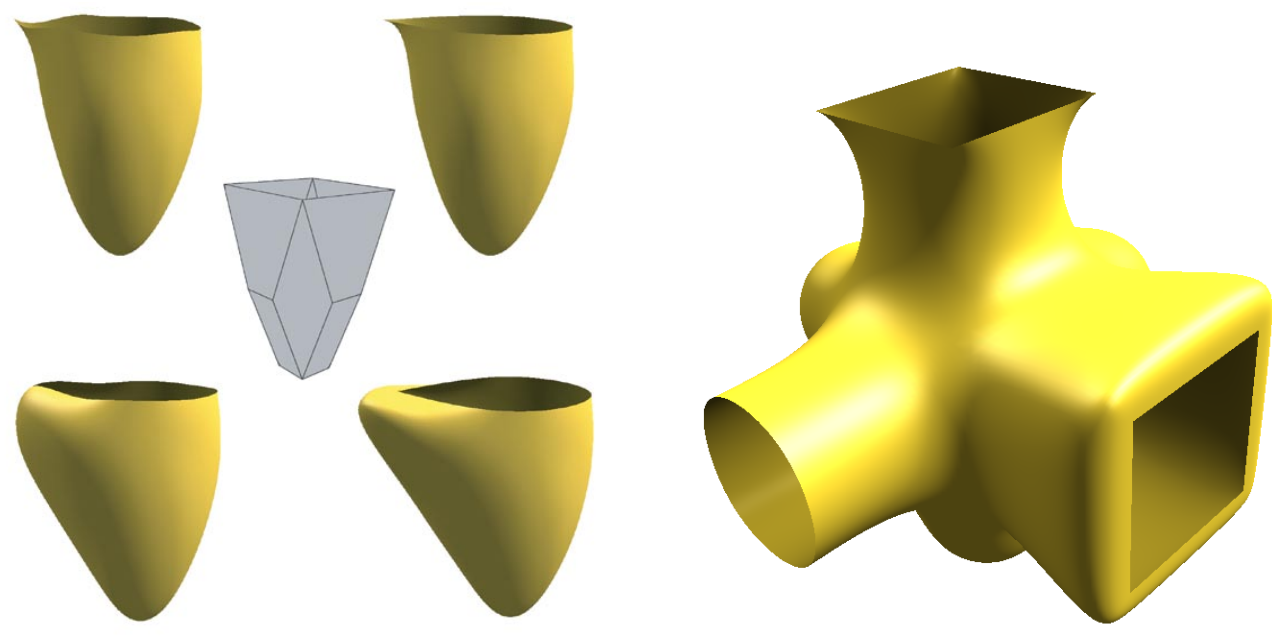

Figure 3: Left: Comparison of corner behavior in the case of interior-dependent and independent boundaries. Right: An example of a $C^{3}$ surface with smooth boundary, convex and concave corners

\section{4. $C^{\infty}$ Manifold Surfaces}

\section{1. $C^{\infty}$-continuous surfaces without Boundaries}

We start with a brief review of the manifold-based construction of Ying and Zorin (2004a), which our construction extends. For a control mesh, this construction is based on defining a set of planar charts, depending only on mesh connectivity, local smooth geometry functions on each chart, and a way to blend all local functions together.

A set $M$ (in our case, the control mesh) has $2 \mathrm{D}$ manifold structure, if a collection of charts $\left(C_{i}, \varphi_{i}\right)$ is defined, where $C_{i}$ are open domains in the plane and $\varphi_{i}$ are one-to-one maps $C_{i} \rightarrow M$, such that the images $\varphi_{i}\left(C_{i}\right)$ cover all of $M$ (we define charts for a mesh $M$ below). $M$ is a $C^{\infty}$ (or $C^{d}$ ) manifold if the transition maps from chart to chart $t_{j i}=\varphi_{j}^{-1} \circ \varphi_{i}$, defined for pairs of charts for which $\varphi_{i}\left(C_{i}\right)$ and $\varphi_{j}\left(C_{j}\right)$ intersect, are $C^{\infty}\left(\right.$ or $\left.C^{d}\right)$.

Ying and Zorin (2004a) construct functions $f_{i}^{l o c}: C_{i} \rightarrow R^{3}$ defining the geometry embedding functions locally on each chart. Then partition of unity functions $w_{i}$ (i.e. functions with support restricted to individual charts $C_{i}$ and summing up to 1) are used to combine local geometry embeddings. On $M$, the complete surface is defined by $\sum_{i}\left(w_{i} f_{i}^{l o c}\right) \circ \varphi_{i}^{-1}$. However, in practice it is evaluated on individual charts $C_{i}$ via

$$
f_{i}(x)=\sum_{j: \varphi_{i}(x) \in \varphi_{j}\left(C_{j}\right)} w_{j}\left(t_{j i}(x)\right) f_{j}^{l o c}\left(t_{j i}(x)\right)
$$

Figure 4 illustrates the construction. All three components are designed to be $C^{\infty}$ to guarantee that the function $f_{i}(x)$, i.e. the resulting surface is $C^{\infty}$, and can be evaluated using closed-form expressions (polynomials and trigonometric functions).

Charts and Transition Maps. Chart maps $\varphi_{i}$ are defined per vertex. Each chart domain is a curved star shape $C_{i}$, obtained by mapping the ring of faces sharing vertex $v_{i}$ to the plane as described below. The overlap between the images of two charts in the mesh is a pair of faces. Instead of constructing the maps $\varphi_{i}$, the maps $\varphi_{i}^{-1}$ are constructed. The chart construction proceeds in two steps: first, the faces adjacent to a given vertex are mapped piecewise bilinearly to the plane (maps $L_{i}$ to domains $S_{i}$ ). Then a transformation 


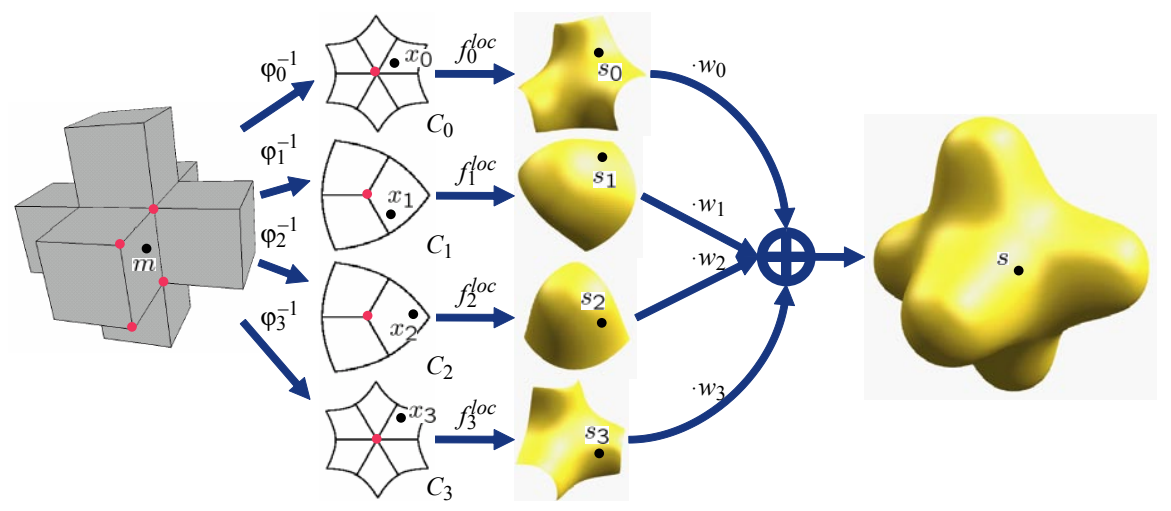

Figure 4: Components of the manifold-based surface construction.

$c_{i}$ is applied to each wedge of the regular star $S_{i} ; c_{i}$ deforms it so that it becomes a conformal image of a square. Therefore, the transition maps are compositions of two functions (Figure 7):

$$
\varphi_{i}^{-1}=c_{i} \circ L_{i}
$$

The map $c_{i}$ is another composition of two maps, a linear map $l_{K_{i}}$ and a conformal map $g_{K_{i}}$ :

$$
c_{i}=g_{K_{i}} \circ l_{K_{i}}
$$

where $g_{K_{i}}=z^{\left(4 \alpha / K_{i}\right)}, z=x+i y$, and

$$
l_{K_{i}}=\left(\begin{array}{cc}
\frac{\cos (\alpha \pi / 4)}{\cos \left(\pi / K_{i}\right)} & 0 \\
0 & \frac{\sin (\alpha \pi / 4)}{\sin \left(\pi / K_{i}\right)}
\end{array}\right) .
$$

$\alpha$ is 1 for charts away from the surface boundary. Other values of $\alpha$ are used for constructing boundary charts.

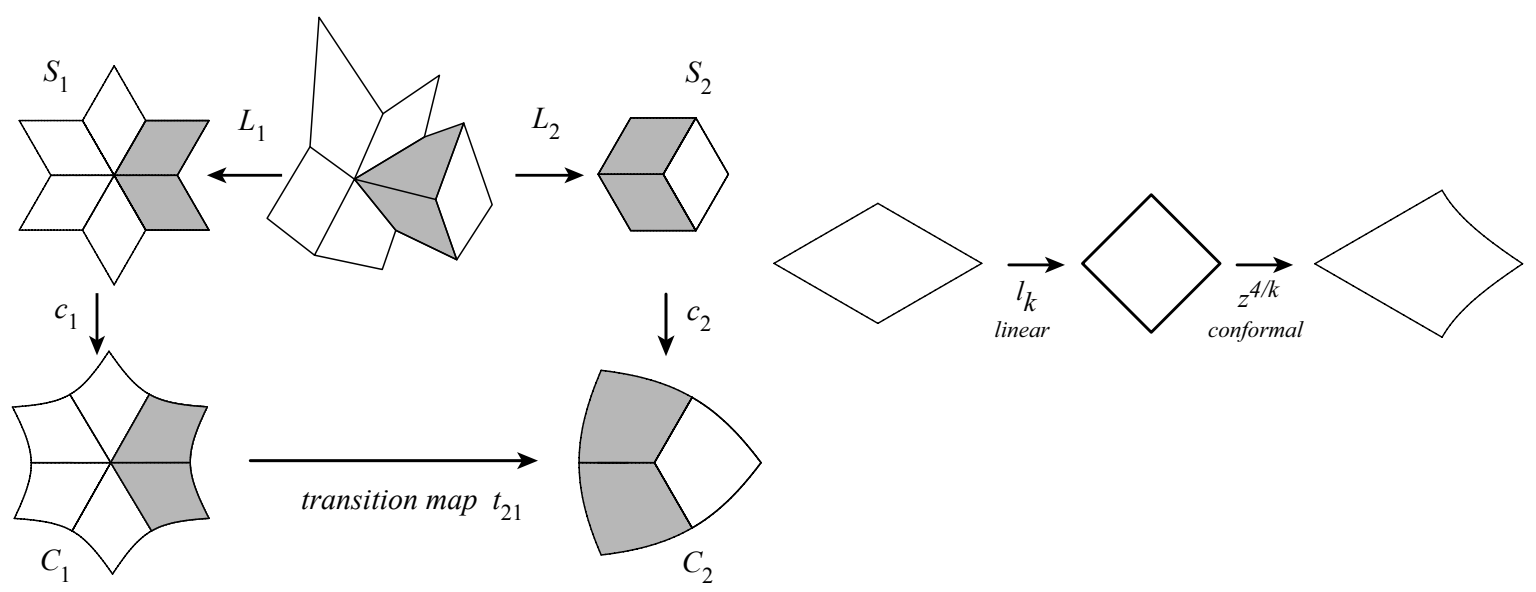

Figure 5: Chart map construction.

Note that only the bilinear maps $L_{i}$ depend on the mesh geometry, and the transition maps $t_{j i}$ depend only on the compositions $L_{j} \circ L_{i}^{-1}$. While each of the maps $L_{i}$ and $L_{j}$ depends on mesh geometry, their composition (which is also bilinear on each face) depends only on the shape of $S_{i}$ and $S_{j}$ which do not depend on mesh geometry. We conclude that $f_{i}$ depends on mesh geometry only through the local geometry functions $f_{j}^{l o c}$. 
Partition of Unity. The partition of unity is built from identical pieces defined initially on the standard square $[0,1]$ as a product of two identical one-dimensional $C^{\infty}$ functions $\eta(u) \eta(v)$. The function $\eta$ can be chosen in different ways, and the choice has a significant impact on surface behavior. We discuss possible choices in detail in Section 6. Ying and Zorin (2004a) uses $\eta$ from Bruno and Kunyansky (2001):

$$
\eta(t)= \begin{cases}1 & : 0 \leq t \leq \delta \\ \frac{h((t-\delta) / a)}{h((t-\delta) / a)+h(1-(t-\delta) / a)} & : \delta<t<1-\delta \\ 0 & : 1-\delta \leq t \leq 1\end{cases}
$$

where $\delta>0$ (1/8 is used), $a=1-2 \delta$ and $h(s)=\exp (2 \exp (-1 / s) /(s-1))$. Once the function is defined on the square, a rotation by $\pi / 4$ combined with the map $g_{K}^{-1}$ is applied to remap $\eta(u) \eta(v)$ to a single wedge. Then the function is defined by rotational symmetry on the rest of the chart.

Defining Geometry. In Ying and Zorin (2004a) geometry is defined using polynomials. Two steps of CatmullClark subdivision are applied to the mesh to define the coarse shape of the surface and then polynomials are used to fit the shape to these data points in the least squares sense. Every vertex of the refined mesh after the subdivision step can be assigned to points with bilinear coordinates $(l / 4, n / 4)$ in each sector of the star $S$ (we omit the subscripts referring to the chart index). For each vertex $v$, these points in $S$ are remapped to the chart domain $C$ using the map $c$. There are $m=12 K+1$ sample points in the interior of $C$, which are denoted $x_{0}, \ldots, x_{m-1}$ (Figure 7 ). The $3 D$ subdivision surface positions for these points are denoted as $s_{0}, \ldots, s_{m-1}$. The polynomial geometry function $f^{l o c}$ of total degree $\leq d=\lfloor\min (14, K+2)\rfloor$ is defined by minimizing the differences $f\left(x_{i}\right)-s_{i}$ in the least squares sense. The choice of 14 as the maximal polynomial degree resulted from experiments with high valence vertices; higher degrees result in small-scale surface artifacts (see Figure 6); reducing the degree makes it impossible to match the overall smooth shape of a subdivision surface.
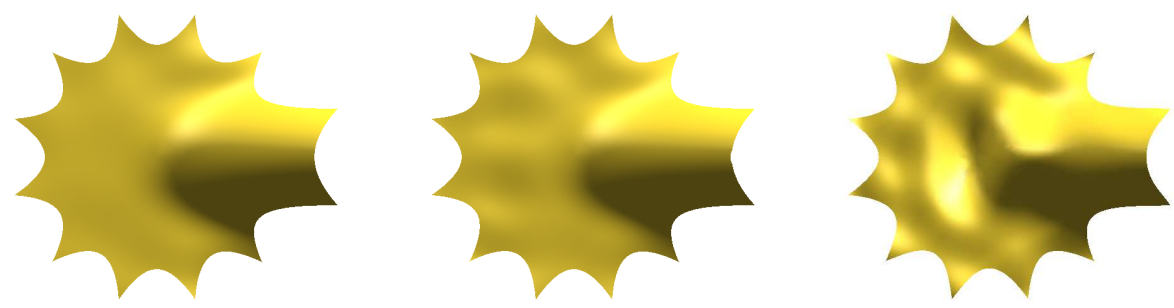

Figure 6: A valance 12 vertex chart with a lifted point on the right represented by polynomial fits of degree 14,15 , and 16 respectively.

As this optimization problem leads to a linear system of equations for polynomial coefficients, the matrix $W$ mapping sample points $s$ to polynomial coefficients depends only on the central vertex valence and can be precomputed for efficient geometry evaluation. For the fitting process, monomials $p_{0}, \ldots, p_{n-1}$ where $n=d(d+1) / 2$ are used as basis functions. A least squares fit is used to solve for the basis coefficients $a_{j}$ such that $f=\sum_{j=0}^{n-1} a_{j} p_{j}$. Let $a$ be the vector of coefficients $a_{j}, s$ be the vector of values $s_{i}$ and $B$ be the $m$ $\mathrm{x} n$ matrix of monomials $p_{j}\left(x_{i}\right)$. Then the vector of coefficients $a$ minimizing $\|B a-s\|^{2}$ can be computed as

$$
a=W s=B^{+} s=\left(B^{T} B\right)^{-1} B^{T} s,
$$

where $B^{+}$denotes the pseudo-inverse of $B$. In our construction, we use a similar approach, however, additional constraints need to be imposed for the boundary.

One can observe that the formula (3) does not guarantee translation invariance by construction, which requires that the rows of $W$ sum up to one. In practice, we observe the rows of $W$ sum up to 1 with high degree of accuracy, but it is desirable to guarantee translation invariance precisely. For this reason, we use a slightly altered formula for $a$ : we set $a_{0}$, the coefficient of the constant monomial $p_{0} \equiv 1$, to $s^{c}$, the position 
of the central sample point of the chart, and fit the remaining monomials to the differences $s-s^{c}$. The vector of coefficients $a^{d}$ with $a_{0}$ excluded is computed as

$$
a^{d}=W\left(s-\bar{s}^{c}\right)
$$

where $W$ is redefined to be pseudoinverse of $B^{\text {diff }}$, with $B_{i j}^{\text {diff }}=p_{j}\left(x_{i}\right), j=1 \ldots n-1$, and $\overline{s^{c}}$ is a vector of the same length as $a$ with all components equal to $s^{c}$, the sample point in the center of the chart.

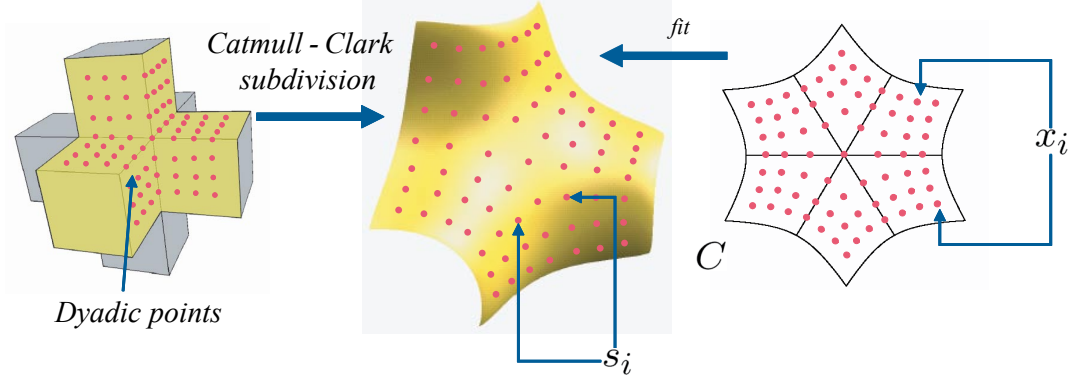

Figure 7: Defining geometry functions.

Summary. To define a manifold surface, we choose chart maps $\varphi_{i}$, geometry maps $f_{i}^{l o c}$ and functions $\eta$ determining partition of unity functions $w_{i}$.

We add more types of charts $\left(C_{i}, \varphi_{i}\right)$ to handle surfaces with boundaries, and consider different choices for $f_{i}^{l o c}$ and $\eta$ in Sections 5 and 6 respectively.

\section{2. $C^{\infty}$ Surfaces with Boundary}

We extend the construction to surfaces with piecewise smooth boundary reusing most elements. The input to our construction is a quadrilateral mesh, with tags for convex and concave corners.

The charts and transition maps for boundary vertices are constructed using star-shaped areas shown in Figure 2, obtained by joining together $k$ equal sectors for a vertex of valence $k$. However, instead of choosing the angular size of each sector to be $2 \pi / k$, we use $\pi / k$ for smooth boundary vertices, $3 \pi /(2 k)$ for concave corners and $\pi /(2 k)$ for convex corners. The formulas for the maps are obtained by choosing $\alpha$ to be $1 / 2$, $3 / 4$ and $1 / 4$ respectively in (2). The partition of unity functions are constructed per quad, in the same way it is done in the interior vertex case.

The most significant difference is in the geometry definition. As discussed in Section 3, one can either constrain the boundary curves to be dependent only on the boundary control points, or allow boundary curves to be influenced by arbitrary points. In both cases, we start with two steps of Catmull-Clark subdivision, as in the interior case. The number of control points is now $m=12 k+5$ instead of $m=12 k+1$, as shown in Figure 8. We use the modified Catmull-Clark scheme described in Biermann et al. (2000a) to handle all types of boundary vertices.

Interior-depedent boundary. The local geometry function is computed as in the interior case using a polynomial fit with a precomputed matrix $W$. We use monomials of total degree $\leq d=\lfloor\min (14, k+2)\rfloor$ as before, except in two empirically determined cases: for a smooth boundary vertex with valance $k=2$ (valence for regular meshes with boundary) we choose $d=6$, and for concave corner vertices with valance $k \geq 6$ we choose degree $d=\max (14, k)$.

Independent boundary. In this case, we proceed in two steps: first, we compute the matrix $W^{\text {bnd }}$ for fitting polynomials only to the boundary points obtained by Catmull-Clark subdivision. Next, we compute a matrix to determine the coefficients of the remaining monomials in the basis, with boundary monomial coefficients fixed.

The smooth boundary vertices and corner vertices have to be treated differently in the first step, because in the case of corners the boundary curve consists of two polynomial pieces. We assume that for a smooth 

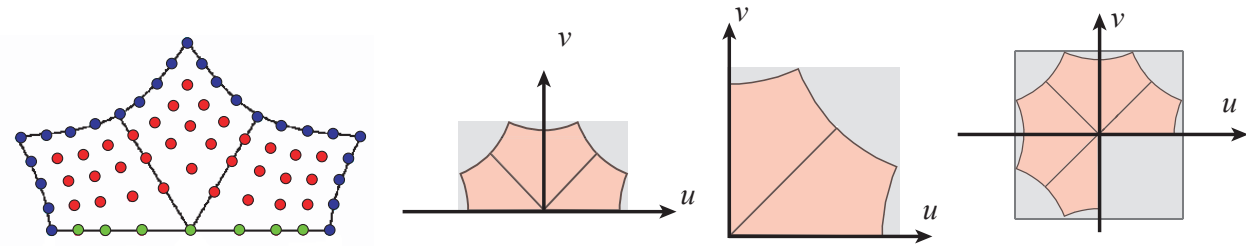

Figure 8: Left: $12 k+4$ sample points for a smooth boundary chart are shown in red (interior points) and green (boundary points). Blue points are not included. Right: coordinate systems for boundary charts.

boundary chart the boundary is aligned with the $u$ axis, and for the corner chart the two boundary pieces are aligned with $u$ and $v$ axes respectively, with the corner at zero. The sample points $x_{i}$ have coordinates $\left(u_{i}, v_{i}\right), i=1 \ldots m$.

For a smooth boundary chart, we define the set of boundary monomials $P_{i}^{b n d}(u, v), i=1 \ldots n^{b n d}$, to be the monomials $u^{i-1}$ with $n^{b n d}=d+1$. For a corner chart (either convex or concave), $n^{\text {bnd }}=2 d+1$, $P_{i}^{b n d}(u, v)=u^{i-1}, i=1 \ldots d+1$, and $P_{i}^{b n d}(u, v)=v^{i-d-1}, i=d+2 \ldots 2 d+1$. The remaining monomials up to total degree $d$, are denoted $q_{j}, j=1 \ldots n-n_{b n d}$. We also split the sample points into two groups: $x_{i}^{b n d}$, $i=1 \ldots 7$, and $x_{i}^{i n t}, i=1 \ldots m-7 . s_{i}^{b n d}$ and $s_{i}^{i n t}$ are corresponding 3D points obtained by subdivision.

The complete geometry function has the form

$$
f^{l o c}(u, v)=\sum_{i=1}^{n_{b n d}} a_{i}^{b n d} P_{i}^{b n d}(u, v)+\sum_{i=1}^{n-n_{b n d}} a_{i}^{i n t} P_{i}^{i n t}(u, v)
$$

Let $B^{r, s}$ be the matrix $\left[p_{i}^{r}\left(x_{j}^{s}\right)\right]$ of the values of monomials evaluated at sample points, with $r, s$ being one of bnd and int.

We first obtain the coefficients $a_{i}^{b n d}$ by minimizing the difference $\left\|B^{b n d, b n d} a^{\text {bnd }}-s^{b n d}\right\|$ between the polynomial boundary curve and sample values $s^{b n d}$; as the number of polynomials typically exceeds the number of sample points on the boundary, the solution is not unique. We use the standard approach to under-constrained optimization and minimize the sum of the squares of the coefficients to obtain a unique solution. This solution is given by $\left(B^{b n d, b n d}\right)^{+} s^{b n d}=W^{b n d} s^{b n d}$. To compute (a regularized) inverse of the possibly singular matrix $\left(B^{b n d, b n d}\right)^{T}\left(B^{b n d, b n d}\right)$, needed for $\left(B^{b n d, b n d}\right)^{+}$, we use SVD regularization. For a matrix $A=U S V^{T}$, the regularized inverse is computed as $U S^{\prime} V^{T}$, where $S^{\prime}$ is a diagonal matrix with entries $S_{i i}^{\prime}=1 / S_{i i}$ for $\left|S_{i i}\right|>\epsilon$, and 0 otherwise. We use $\epsilon=10^{-10}$.

Note that this procedure works as well for smooth and corner boundary cases. In the corner boundary case, it is equivalent to fitting polynomials in $u$ to the segment of the boundary aligned with the $u$ axis, and polynomials in $v$ to the segment of the boundary aligned with the $v$ axis. The point samples on the boundary are interpolated, as the degree of the polynomials we use always exceeds the number of points. One could entirely eliminate the fitting step, and simply interpolate the points with sufficiently low degree polynomials, setting the coefficients for higher-order monomials to zero; however, using all polynomials up to degree $d$ and minimizing the norm of coefficients appears to produce better results.

We determine the remaining coefficients $a^{i n t}$ by minimizing $\sum_{i=8}^{m}\left(f^{l o c}\left(x_{i}\right)-s_{i}\right)^{2}$, while keeping the boundary coefficients $a_{i}^{\text {bnd }}$ fixed. The matrix form of this expression is $\left\|B^{\text {int, int }} a^{\text {int }}+B^{\text {int, bnd }} a^{\text {bnd }}-s^{\text {int }}\right\|^{2}$, which leads to the following expression for $a^{i n t}$ :

$$
a^{i n t}=\left(B^{i n t, i n t}\right)^{+}\left(s^{i n t}-B^{i n t, b n d} a^{b n d}\right)
$$

To sum up, the vector of coefficients $a$ is computed from the vector of sample points $s$ as

$$
a=\left(\begin{array}{l}
a^{i n t} \\
a^{\text {bnd }}
\end{array}\right)=\left(\begin{array}{ll}
\left(B^{\text {int,int }}\right)^{+} & -\left(B^{\text {int,int }}\right)^{+} B^{\text {int, }, \text { bnd }}\left(B^{\text {bnd,bnd }}\right)^{+} \\
0 & \left(B^{\text {bnd,bnd }}\right)^{+}
\end{array}\right)\left(\begin{array}{l}
s^{\text {int }} \\
s^{\text {bnd }}
\end{array}\right)=W s
$$

where $W$ depends only on the valence of the center of the chart and can be precomputed. 


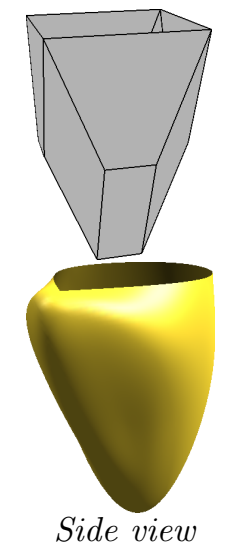

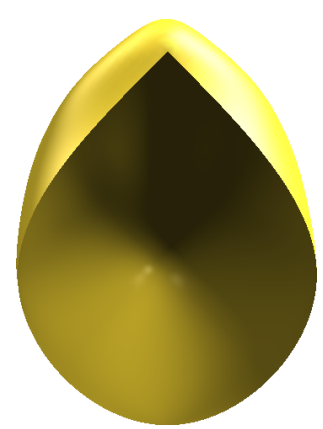

Catmull-Clark

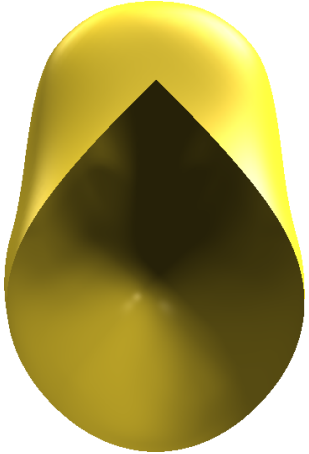

$C^{\infty}$ surface

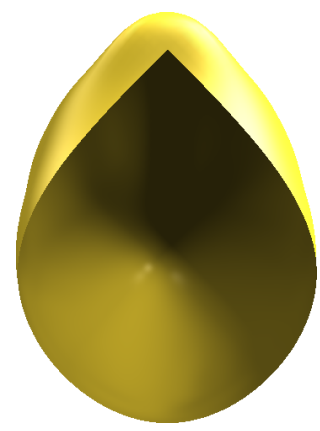

$C^{3}$ surface

Figure 9: Limitation of concave corners with independent boundary: Catmull-Clark surface with corners cannot be approximated well by $C^{\infty}$ surfaces; a much closer approximation can be obtained with spline-based $C^{k}$ surfaces.

Limitation of the interior-independent construction.. For concave corners and interior-independent boundaries, the construction described above may produce somewhat undesirable shapes, independently of the parameters of the subdivision scheme used to compute the points $x_{i}$. Specifically, in cases when a concave corner is prescribed for a control mesh vertex for which the plane formed by the boundary edges meeting at the vertex is close to perpendicular to the nearby mesh faces (See Figure 9). In this case, even if the underlying subdivision surface makes a sharp turn to approach the boundary curve from the outside, as it is necessary for a concave corner, the polynomial approximation deviates significantly from the surface, and "overshoots" in a significant way.

The reason for this behavior can be understood as follows. Consider the curve on the local surface corresponding to line $u=0$ in the chart. Note that all polynomials $P_{i}^{i n t}$ vanish, so this curve on the surface is completely determined by the expression $\sum_{i} a_{i}^{b n d} P_{i}^{b n d}(0, v)$; as the coefficients $a_{i}^{b n d}$ are computed using boundary data only, the points $s_{i}^{i n t}$ have no effect on the curve behavior, even if it passes through corresponding points $s_{i}^{i n t}$ in the interior of the surface. This is in contrast to the interior-dependent construction, which results in better behavior.

To a large extent, this problem can be addressed by using $C^{d}$-continous surfaces based on splines, as explained in the next section (See Figure 9).

\section{5. $C^{d}$-continuous Surfaces}

While for $C^{\infty}$ surfaces derivatives of parametrizations are formally defined for all orders, their magnitudes of these derivatives can grow rapidly. There is a lower bound for this growth: for linear parametric surface constructions with localized influence of control points it is impossible to have an upper bound for parametrization derivatives uniform in the derivative order (see Section 6). It turns out that derivative magnitudes can be reduced and better surface quality can be achieved if we consider $C^{d}$-continuous surfaces for some finite $d$, constructed using splines instead of polynomials both for geometry and partition-of-unity functions (Figure 10)).

\section{1. $C^{d}$ Construction for Interior Charts}

The $C^{\infty}$ and $C^{d}, d<\infty$, constructions are similar for interior vertices. Charts and transition maps remain the same as for $C^{\infty}$ construction. For $C^{d}$-continuity, we replace the 1D partition of unity functions $h$ with a polynomial function (Section 6$)$. We use a uniform bi-degree $(d+1)$ tensor product B-Spline fit for the geometry.

Similar to the $C^{\infty}$ construction, we start with two steps of modified Catmull-Clark subdivision to get $m$ points $x_{0}, \ldots, x_{m-1}$ in the domain $C$ (Figure 7 ) and corresponding surface points by $s_{0}, \ldots, s_{m-1}$

We found it important to include an extra ring of points on the subdivision surface corresponding to the outer boundary of the chart domain to ensure a better match between adjacent patches. Thus, we have 


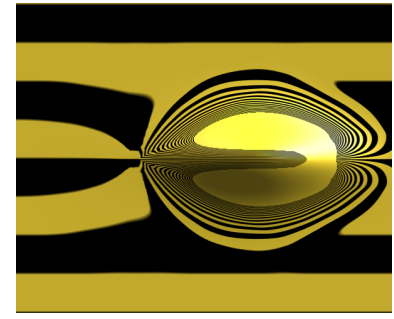

Catmull-Clark

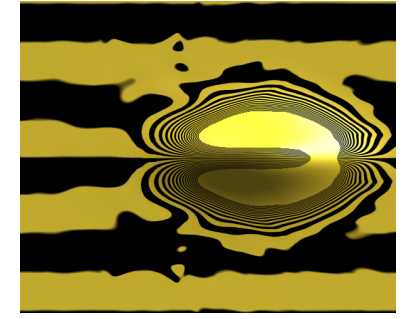

$C^{\infty}$ surface

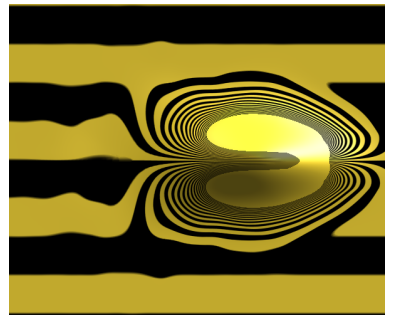

$C^{3}$ surface, $g=5$.

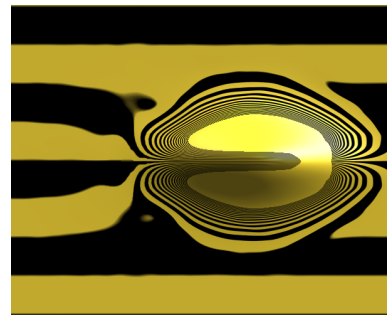

$C^{3}$ surface, $g=7$.

Figure 10: A flat mesh with a single extraordinary vertex of valence 12 . The regular vertex adjacent to valence 12 vertex is lifted. $C^{k}$ spline-based surfaces can closely match the reflection line pattern for the subdivision surface, although the match is not perfect due to larger area influenced by each vertex.

$m=20 K+1$ for interior charts and $m=20 K+5$ for smooth boundary and corner charts, where $K$ is the valence of the vertex.

The domain of our spline surface is the minimal box containing sample points $x_{0}, \ldots, x_{m-1}$ (Figure 11). For the geometry function defined using splines, in addition to the degree, we can also choose the number of spline patches for the domain. We use a $g \times g$ grid, uniformly spaced in both directions on this domain, separating it into $(g-1) \times(g-1)$ patches. The case of $g=2$ is essentially identical to the case of monomial basis functions used for the $C^{\infty}$ construction. Experimentally, we have observed that increasing $g$ above 3 does not have any significant advantages for interior points, but leads to improvements for smooth boundaries and concave corner points. We note that increasing $g$ results only in a modest additional cost for surface evaluation, as it is primarily determined by the degree of splines, not the number of patches.

We use coordinates $(u, v)$ in the range $[0 \ldots g-1]$ in each direction, scaling the grid of sample points so that the domain is square. The center of the chart corresponds to the point $((g-1) / 2,(g-1) / 2)$. We choose the axis direction so that one of them is aligned with an edge direction in the chart. The choice of the edge is arbitrary, and the resulting surface does depend on the way this alignment is chosen for valences which are not multiples of 4 . However, we did not observe any significant effects on either surface visual appearance or parametrization derivative behavior. For a spline of degree $d+1$ (and continuity $C^{d}$ ), $n_{c}=g+d$ control points are needed in each direction. The geometry function is given by

$$
f(u, v)=\sum_{j=0}^{n_{c}-1} \sum_{k=0}^{n_{c}-1} a_{j k} N_{j}(u) N_{k}(v)
$$

where $a_{j k}$ are the control points. In many cases, it is convenient to use a single index for the spline control points $a_{j k}$ and basis functions $N_{j}(u) N_{k}(v)$. We define $a_{\left(j n_{c}+k\right)}=a_{j k}$ and $B_{\left(j n_{c}+k\right)}(u, v)=N_{j}(u) N_{k}(v)$. Then $f(u, v)$ can be compactly written as $a^{T} B(u, v)$, where $a$ and $B(u, v)$ are vectors of length $n_{c}^{2}$.

The fit for spline patches proceeds similarly to the $C^{\infty}$ surface construction. An important change essential for spline surfaces is regularization of the fit, especially for higher values of $g$ : the support of basis functions centered away from the chart center may overlap few if any sample points. We use the thin-plate energy $\int\left(\partial_{u u} f\right)^{2}+2\left(\partial_{u v} f\right)^{2}+\int\left(\partial_{v v} f\right)^{2}$ for regularization: the energy can be written as $a^{T} R^{i n t} a$, where $R^{i n t}$ is a constant matrix. This energy ensures that in areas where there are no sample points the function $f(u, v)$ is as close to linear as possible.

We construct the matrix $B$ of basis functions evaluated at sample points, $B_{i l}=B_{l}\left(u_{i}, v_{i}\right)$, where $i=$ $0 \ldots m-1$ and $l=0 \ldots n_{c}^{2}-1$. The matrix $W$ is obtained as regularized pseudoinverse of $B$ :

$$
W=\left(\left(B^{T} B+\epsilon_{i n t} R^{i n t}\right) B^{T}\right)^{-1}
$$

The choice of $\epsilon_{i n t}$ and regularization parameters for all other cases is discussed in Section 5.4.

To ensure translation invariance, we fit the differences between sample point positions $s_{i}$ and the central sample position $s^{c}$, as we did for the $C^{\infty}$ construction. The monomial $p_{0} \equiv 1$ is no longer a basis function, so we still retain a full matrix $B$ in the fit. We observe that if we shift the sample positions $s_{i}$ by $s^{c}$, so that the central sample position becomes 0, apply the fit, and shift them back, resulting surface is 
translation-invariant thanks to the standard spline property $\sum_{l} B_{l}(u, v)=1$. This allows us to define the spline coefficients as

$$
a=W\left(s-\bar{s}^{c}\right)+\bar{s}^{c}
$$

where as before $\bar{s}^{c}$ is a vector of the same length as $a$ with all components equal to $s^{c}$, the sample point in the center of the chart.

\section{2. $C^{d}$ Surfaces with Boundary}

We do not discuss the interior-dependent boundary construction in detail, as it is identical to the interior chart case, and focus on the interior-independent boundary construction. We use a grid of knots defined in the same way as for the interior charts.

For a smooth boundary chart, we assume that the parametric line corresponding to the surface boundary coincides with the $u$ axis, with the chart center $x^{c}=\left(u^{c}, v^{c}\right)=(t, 0)$, with $t=(1 / 2)(g-1)$. (Figure 11). For a convex corner, two perpendicular sides of the chart are on $u$ and $v$ axes with the corner at $(0,0)$ and the concave corner chart is centered at $(t, t)$ as it was the case for the interior chart. (See Figure 11).
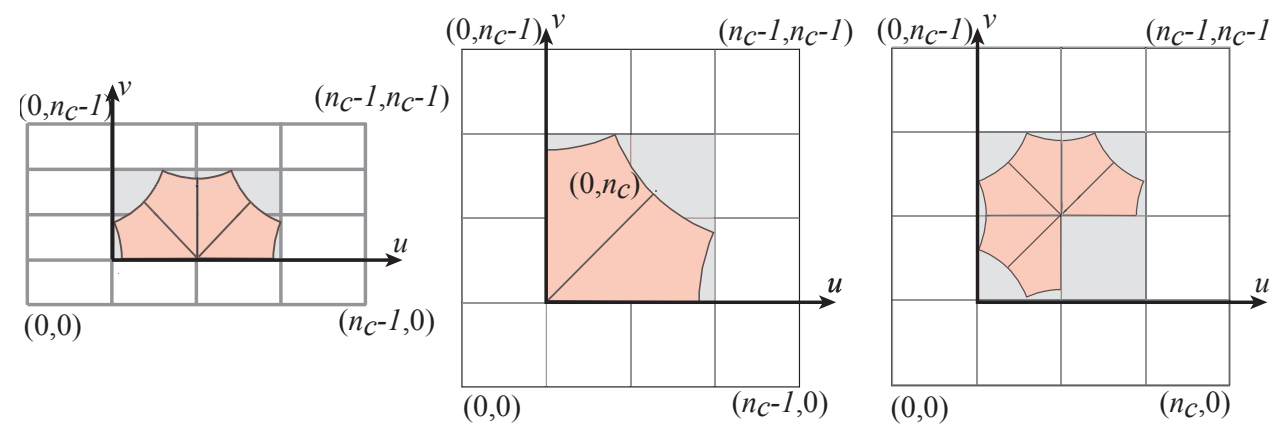

Figure 11: Knot numbering and patch layout for smooth boundary, convex and concave charts for $g=3$.

As before, we need to define a matrix $W$, depending on sample points $x_{i}=\left(u_{i}, v_{i}\right)$ and the degree of the basis, which allows us to compute the vector of spline control points $c$ from the vector of sample positions $s$ as in (7). We split the vector of sample positions $s$ into two subvectors: $s=\left[s^{i n t}, s^{b n d}\right]$, where $s^{\text {bnd }}$ is the vector of 9 boundary sample positions and $s^{i n t}$ is the vector of $m-9$ interior positions.

Our construction consists of two steps similar to the $C^{\infty}$ case:

1. First, we compute the matrix $W^{b n d}$ mapping the boundary sample positions $s^{b n d}$ to the control points $\bar{a}$ for the boundary curves. For the corner charts, we additionally constrain the corner point to be interpolated.

2. In the second step, we use the curve control points $\bar{a}$ computed in the first step to define constraints on the surface control points $a$, and compute the constrained least-squares fit matrix mapping the curve control points $\bar{a}$ and interior sample positions $s^{i n t}$ to the control points of the spline $a$.

The composition of matrices obtained at two steps yields $W$.

Before we proceed with the details of the construction for smooth boundaries and two corner types, we introduce additional notation.

Constrained least-squares fit matrices. In several cases in our construction, we need to compute a matrix arising from a regularized constrained least-squares fit:

$$
x^{T} R x+\|Q x-r\|^{2} \rightarrow \min , \text { subject to } C x=h .
$$

where $Q$ is a $n \times m$ matrix, and $C$ is $r \times n$ matrix of constraints for some $r<n, R$ is a regularization matrix.

Solving this problem with Lagrange multipliers $y$ leads to the system

$$
\left(\begin{array}{cc}
2 Q^{T} Q+R & B^{T} \\
B & 0
\end{array}\right)_{11}\left(\begin{array}{l}
x \\
y
\end{array}\right)=\left(\begin{array}{c}
2 Q^{T} r \\
h
\end{array}\right)
$$


The solution of this system can be written as

$$
x=L_{r}(Q, C, R) r+L_{c}(Q, C, R) h
$$

where $L_{r}(Q, C, R)$ is a $n \times n$ matrix and $L_{c}(Q, C, R)$ is a $n \times m$ matrix. The explicit expressions for the matrices $L_{r}(Q, C, R)$ and $L_{c}(Q, C, R)$ are

$$
\begin{aligned}
& L_{r}(Q, C)=\left(I-Q_{S}^{-1} C^{T} Q_{C}^{-1} C\right)\left(Q_{S} Q^{T}\right)^{-1} \\
& L_{c}(Q, C)=Q_{S}^{-1} C^{T} Q_{C}^{-1}
\end{aligned}
$$

where $Q_{S}=Q^{T} Q+R, Q_{C}=C Q_{S}^{-1} C^{T}$, and the inverses are computed using SVD regularization for stability. This notation allows us to derive the matrices $W$ mapping sample positions to control points in a compact form.

\section{Step 1: Boundary computation}

For smooth vertices, we compute the boundary for the chart by fitting a spline curve of degree $d+1$ to the data points $s_{i}^{b n d}$ corresponding to boundary points $x_{i}^{b n d}$. For corners, we fit two separate curves to the subsets of sample points on two edges of the chart. We also require the corner sample position to be interpolated. In the second step, we ensure that boundary curves of the patch coincide with the curves computed at this step.

As the number of boundary points we fit our curve to is 9 (smooth case) or 5 (for each of the curves in the corner case). If the number of control points for a curve exceeds the number of sample positions we fit to, the least-squares problem is underdetermined. As in the case of polynomial geometry functions, we use SVD-regularized inverses in these cases to obtain a solution.

Smooth boundary charts. For a smooth boundary chart, there are $n_{c}$ control points $\bar{a}_{j}$ of the boundary curve $c(u)$ which we arrange in vector $\bar{a}$ :

$$
c(u)=\sum_{j=0}^{n_{c}-1} \bar{a}_{j} N_{j}(u)
$$

In vector form, the boundary curve $c(u)$ sampled at points $x_{i}^{b n d}, i=0 \ldots 8$, can be written as $B^{b n d, s} \bar{a}$, with

$$
B_{i j}^{b n d, s}=N_{j}\left(u_{i}\right), i=0 \ldots 8, j=0 \ldots n_{c}-1
$$

The control points $\bar{a}$ of the boundary curve are obtained by unconstrained minimization of $\| B^{b n d, s} \bar{a}-$ $s^{b n d} \|^{2}+\epsilon_{b n d} \bar{a}^{T} R^{b n d, s} \bar{a}$, where $R^{b n d, s}$ is the second derivative regularizer matrix for the boundary basis functions $N_{j}(u)$. Minimizing this expression leads to $\bar{a}=W^{b n d} s^{b n d}$, with

$$
W^{b n d}=\left(\left(B^{b n d, s}\right)^{T}\left(B^{b n d, s}\right)+\epsilon_{b n d} R^{b n d, s}\right)^{-1}
$$

i.e., the regularized pseudoinverse of $B^{b n d, s}$.

Convex and concave corner charts. The differences between the way boundary control points are computed for convex and concave corners are minor, and we discuss these together. In both cases we define two boundary curves $c^{u}(u)$ and $c^{v}(v)$.

For a convex corner chart, there are $n_{c}$ control points for each of the boundary directions, and for concave corners, there are $d+\lceil g / 2\rceil=n_{c}^{\prime}$ for each. In the latter case, there are fewer control points because only the part of $c(u)$ and $c(v)$ corresponding to $u^{c} \leq u \leq g-1\left(v^{c} \leq v \leq g-1\right)$ needs to be boundary independent, and these curve segments require fewer control points than the complete boundary curves. We split the vector of sample positions $s^{b n d}$ into three parts, $s^{b n d}=\left[s^{c} s^{u} s^{v}\right]: s^{c}$ is the corner point, and $s^{u}$ and $s^{v}$ are vectors of sample positions along $u$ and $v$ boundary directions. Due to use of (7) for the fit, we can assume $s^{c}$ to be zero, but we do not rely on this assumption in our derivation. Corresponding parametric locations are $x_{i}^{u}=\left(u_{i}^{u}, v^{c}\right), x_{i}^{v}=\left(u^{c}, u_{i}^{u}\right), i=0 \ldots 3$. We assume that these are symmetric, that is, $u_{i}^{u}=v_{i}^{v}$.

Similarly to (10), we define 


$$
\begin{aligned}
& B_{i j}^{b n d, u}=N_{j}\left(u_{i}^{u}\right), i=0 \ldots 3, j=m_{0} \ldots n_{c}-1 \\
& B_{i j}^{b n d, v}=N_{j}\left(v_{i}^{u}\right), i=0 \ldots 3, j=m_{0} \ldots n_{c}-1
\end{aligned}
$$

where $m_{0}=0$ for convex corners and $m_{0}=\lfloor g / 2\rfloor$ for concave corners, so that the number of control points is $n_{c}^{\prime}$.

As the boundary parametric locations are symmetric, we use a single matrix instead:

$$
B^{b n d}=B^{b n d, u}=B^{b n d, v} .
$$

In addition to fitting the curves to $s^{u}$ and $s^{v}$, we need to enforce interpolation of $s^{c}$. The interpolation conditions for the two curves are

$$
\sum_{j=m_{0}}^{n_{c}-1} \bar{c}_{j}^{u} N_{j}\left(u^{c}\right)=s^{c}, \quad \sum_{j=m_{0}}^{n_{c}-1} \bar{c}_{j}^{v} N_{j}\left(v^{c}\right)=s^{c} .
$$

In vector form, $b^{T} \bar{c}^{u}=b^{T} \bar{c}^{v}=s^{c}$ with $b$ defined by

$$
b_{j}=N_{j}\left(u^{c}\right)=N_{j}\left(v^{c}\right)
$$

(Here we take advantage of symmetry again: $u^{c}=v^{c}=t$.)

Now the problem of finding the boundary curve control points $\bar{a}^{u}$ is formulated in the form (8), with $Q=B^{b n d}, C=b^{T}, R=\epsilon_{b n d} R^{b n d, c}$ (the second derivative regularizer for boundary basis functions), $r=s^{u}$, $h=s^{c}$ and $x=\bar{a}^{u}$; this holds for $\bar{a}^{v}$ as well. Using (9),

$$
\begin{aligned}
& \bar{a}^{u}=L_{r}\left(B^{b n d}, b^{T}, \epsilon_{b n d} R^{b n d, c}\right) s^{u}+L_{c}\left(B^{b n d}, b^{T}, \epsilon_{b n d} R^{b n d, c}\right) s^{c}, \\
& \bar{a}^{v}=L_{r}\left(B^{b n d}, b^{T}, \epsilon_{b n d} R^{b n d, c}\right) s^{v}+L_{c}\left(B^{b n d}, b^{T}, \epsilon_{b n d} R^{b n d, c}\right) s^{c},
\end{aligned}
$$

Combining these vectors together into a single vector of boundary control points $\bar{a}=\left[\bar{a}^{u}, \bar{a}^{v}\right]$, we get the following expression for $W^{\text {bnd }}$ in block matrix form:

$$
\bar{a}=W^{b n d} s^{b n d}=\left(\begin{array}{lll}
L_{c} & L_{r} & 0 \\
L_{c} & 0 & L_{r}
\end{array}\right)\left(\begin{array}{l}
s^{c} \\
s^{u} \\
s^{v}
\end{array}\right)
$$

where we have omitted arguments of $L_{r}$ and $L_{c}$. We simplify this expression somewhat using $s^{c}=0$ : the first column in the matrix can be eliminated.

\section{Step 2: Interior control point computation}

We obtain the control points $a_{i j}$ for the surface patch by fitting $f(u, v)$ to the interior sample positions $s_{i}^{\text {int }}$, while keeping the boundary curves fixed.

Smooth boundary chart. In the case of a smooth boundary chart, there is only one boundary curve $f(u, 0)$ and we require that it coincides with the curve obtained at the first step:

$$
f(u, 0)=c^{u}(u)=\sum_{j=0}^{n_{c}-1} \bar{a}_{j}^{u} N_{j}(u)
$$

Rearranging terms, we observe that the boundary curve is a spline curve of degree $d+1$

$$
f(u, 0)=\sum_{j=0}^{n_{c}-1}\left(\sum_{k=0}^{n_{c}-1} a_{j k} N_{k}(0)\right) N_{j}(u)
$$


and it coincides with $c(u)$ if and only if their control points coincide: $\sum_{k=0}^{n_{c}-1} a_{j k} N_{k}(0)=\bar{a}_{j}$, or in matrix form,

$$
M^{s} a=\bar{a}
$$

where the entries of the matrix $M^{s}$ are

$$
M_{j l}^{s}=\left\{\begin{array}{l}
N_{\left(l-j n_{c}\right)}(0), \text { for } j n_{c} \leq l<(j+1) n_{c} \\
0 \text { otherwise }
\end{array},\right.
$$

with $l=0 \ldots n_{c}^{2}-1, j=0 \ldots n_{c}-1$.

The constrained least squares fit problem for the vector of control points $a$ for the patch can now be written as

$$
\epsilon_{s} a^{T} R^{s} a+\left\|B^{\text {int }} a-s^{i n t}\right\|^{2} \rightarrow \min , \text { subject to } M a=\bar{a}
$$

with $B_{i l}^{i n t}=B_{l}\left(u_{i}^{i n t}, v_{i}^{i n t}\right), i=0 \ldots m-10, l=0 \ldots n_{c}^{2}-1$, i.e., is of the form (8).

Using (9), we obtain

$$
a=L_{r}\left(B^{i n t}, M, \epsilon_{s} R^{s}\right) s^{i n t}+L_{c}\left(B^{i n t}, M, \epsilon_{s} R^{s}\right) \bar{a}=L_{r}\left(B^{i n t}, M, \epsilon_{s} R^{s}\right) s^{i n t}+L_{c}\left(B^{i n t}, M, \epsilon_{s} R^{s}\right) W^{b n d} s^{b n d}
$$

or

$$
a=W s=\left(L_{r}\left(B^{i n t}, M, \epsilon_{s} R^{s}\right) L_{c}\left(B^{i n t}, M, \epsilon_{s} R^{s}\right) W^{b n d}\right) s
$$

Convex and concave corner charts. In this case we need to consider two boundary curves, $f\left(u, v^{c}\right)$ and $f\left(u^{c}, v\right)$. For convex corners, we require these curves to coincide with the curves $c^{u}(u)$ and $c^{v}(v)$ along two boundaries of the patch $0 \leq u, v \leq g-1$. For concave corners, only one half of each patch boundary is the surface boundary, so we require $c^{u}(u)=f\left(u^{c}, v\right)$ only for $u^{c} \leq u \leq g-1$. Similar to the smooth boundary, these constraints are equivalent to constraints relating surface control points $a_{j k}$ to the boundary control points:

$$
\bar{a}_{j}^{u}=\sum_{k=0}^{n_{c}-1} a_{j k} N_{k}\left(v_{c}\right), \quad \bar{a}_{k}^{v}=\sum_{j=0}^{n_{c}-1} a_{j k} N_{j}\left(u_{c}\right), \quad j, k=m_{0} \ldots n_{c}-1 .
$$

In matrix form,

$$
\bar{a}^{u}=M^{u} a, \bar{a}^{v}=M^{v} a .
$$

with matrix entries $M^{u}$ and $M^{v}$ defined by

$$
M_{j l}^{u}=\left\{\begin{array}{l}
N_{\left(l-j n_{c}\right.}\left(v_{c}\right), \text { for } j n_{c} \leq l<(j+1) n_{c} \\
0 \text { otherwise }
\end{array}, \quad M_{k l}^{v}=\left\{\begin{array}{l}
N_{\left.l l / n_{c}\right\rfloor}\left(u_{c}\right), \text { for } l \bmod n_{c}=k \\
0 \text { otherwise }
\end{array}\right.\right.
$$

for $j, k=m_{0} \ldots n_{c}-1$ and $l=0 \ldots n_{c}^{2}-1$. Combining the two sets of constraints together, we get

$$
M a=\left(\begin{array}{c}
M^{u} \\
M^{v}
\end{array}\right) a=\left(\begin{array}{c}
\bar{a}^{u} \\
\bar{a}^{v}
\end{array}\right)
$$

Again, we solve a constrained problem of the form (8).

$$
\epsilon_{c} a^{T} R^{c} a+\left\|B^{\text {int }} a-s^{i n t}\right\|^{2} \rightarrow \min , \text { subject to } M a=\bar{a}
$$

with the same matrix $B^{\text {int }}$ for convex corners as for smooth boundary.

This formulation yields formulas for $W$ identical to (18). 


\subsection{Summary of the boundary construction.}

For each chart type (smooth boundary, convex corner, concave corner) and each valence, we precompute a matrix $W$, which depends only on the chart type, valence and the degree of smoothness $d$. At run-time, the control points of the patch are computed from subdivision sample positions $s$ as $a=W s$.

The matrix $W$ is

$$
W=\left(L_{r}\left(B^{i n t}, M, R\right), L_{c}\left(B^{i n t}, M, R\right) W^{b n d}\right)
$$

where $R$ is a second-derivative regularizer matrix. For smooth boundaries, $W^{\text {bnd }}$ is given by (11); for corners, $W^{b n d}$ is given by (15) in terms of $B^{b n d, c}$ and $b$, defined by (10) and (14) respectively.

The constraint matrices $M$ are defined by (17) for the smooth boundary and (20) for convex and concave corners.

\subsection{Choosing regularization parameters.}

We use four regularization parameters: $\epsilon_{b n d}$ for the boundary curve fit, and $\epsilon_{i n t}, \epsilon_{s}$ and $\epsilon_{c}$ for different types of charts. We defined all of these parameters with respect to a a single reference parameter $\epsilon$ setting the overall scale of regularization.

The number of terms $m_{f i t}$ in the least-squares part of the energy we use for fitting is equal to the number of internal sample points with boundary points excluded: $20 K+1$ for interior charts, $20 K-4$ for boundary charts and 9 on the boundary, and we need to scale the regularization term in a similar way so that the ratio between the least-squares fit energy and regularization energy is (approximately) independent of $K$. Because the domain of the spline has size $(g-1) \times(g-1)$, to make the regularization energy independent of the choice of the number of patches, we can rescale it to unit size, yielding a scale factor $(g-1)^{2}$. The combination of these terms leads to the normalization factor $m_{f i t}(g-1)^{2}$ for the regularization term.

We choose all regularization parameters to be of the form $\epsilon_{x}=C_{x} m_{f i t}(g-1)^{2} \delta_{x}$, where the constants $\delta_{x}$ independent of $d, g$ and $K$, are determined experimentally based on quality of the results for basis functions. The results are only moderately sensitive to the changes in regularization parameters, so we determine them up to an order of magnitude. Most regularization is required for concave corner vertices, for which the fit to the subdivision surface sample points is least accurate. The constants we use are shown below.

\begin{tabular}{|c|c|c|c|c|}
\hline$\epsilon_{\text {bnd }}$ & $\epsilon_{\text {int }}$ & $\epsilon_{s}$ & $\epsilon_{c, \text { convex }}$ & $\epsilon_{c, \text { concave }}$ \\
\hline $10^{-7}$ & $10^{-7}$ & $10^{-6}$ & $10^{-6}$ & $10^{-4}$ \\
\hline
\end{tabular}

\section{Partition of Unity Functions}

One of the reasons to consider the $C^{d}$ construction described in Sections 5.1 and 5.2 is to obtain better behavior for lower-order derivatives than we get for the $C^{\infty}$ construction. Numerical estimates show that the magnitude of the derivatives is primarily determined by the magnitude of the derivatives of partition of unity (POU) functions $w_{i}$ in (1), rather than chart maps or geometry functions. As our POU functions are obtained as tensor products of one-dimensional POU functions, the magnitude of their derivatives can be estimated using derivatives of these one-dimensional functions.

The POU function $h$ from Bruno and Kunyansky (2001) used in the $C^{\infty}$ construction is designed to be $C^{\infty}$-continuous, but no attempt was made to optimize its derivative magnitudes. One of the difficulties in using an optimization approach in $C^{\infty}$ setting is that the solutions of suitable optimization problems tend to be in a space of functions of finite smoothness. This is not an obstacle for $C^{d}$ surfaces, as long as POU functions have smoothness $d$ or higher. However, there are fundamental limitations to what can be achieved, because of the fixed support of the basis functions used in our construction. In particular, we consider the optimal (in the sense of minimal sup-norm of derivatives) POU function (a perfect spline), and observe that it still has relatively fast derivative growth.

To simplify notation, in the remainder of this section we consider $C^{d-1}$ rather than $C^{d}$ surfaces, so that $d$ corresponds to the degree of the polynomials used to construct some of the POU functions. Given any $C^{d-1}$ function $\xi$, which we call blending function, satisfying

$$
\xi^{(i)}(0)=\xi^{(i)}(1)=0, i=1 \ldots d-1 ; \xi(0)=0, \xi(1)=1,
$$


we obtain a $C^{d-1}$-continuous partition of unity function for surfaces in the same way as in Section 4.1. The partition of unity property is guaranteed if $\xi(u)$ satisfies the natural symmetry condition

$$
\xi(u)=1-\xi(1-u)
$$

In addition to $C^{\infty}$ POU functions, we consider three more types of POU functions; all these functions are piecewise-polynomial:

- Uniform spline of degree $d$, satisfying boundary conditions (22).

- POU function minimizing the $L^{2}$-norm of $d$-th derivative ( $W_{d}^{2}$ norm).

- POU function minimizing the sup norm of $d$-th derivative ( $W_{d}^{\infty}$ norm).

For most geometric modeling applications, minimizing sup norm is likely to be most relevant. However, as we demonstrate, the behavior of piecewise polynomial functions is qualitatively similar for practical values of $d$.

Uniform spline blending function. This is the simplest $C^{d-1}$ POU function to define. We use a sequence of $2 d+1$ knots $0,1 / d, 2 / d \ldots 2(d-1) / d, 2 d$, with $d$ control values all set to 1 , that is,

$$
\xi_{\text {unif }, d}(u)=\sum_{i=0}^{d-1} B^{d}(d u-i)
$$

where $B^{d}(u)$ is the B-spline basis function of degree $d$ for knot spacing 1 , supported on $[0, d+1]$.

As $\xi_{\text {unif,d }}(u)$ is $C^{d-1}$ by construction, and for $u<0 \xi(u) \equiv 0$, the boundary conditions (22) are satisfied at $u=0$. Using the standard spline partition-of-unity property, $\sum_{i=-d}^{d-1} B^{d}(d u-i) \equiv 1$ on $[0,1]$, and spline basis function symmetry $B^{d}(u)=B^{d}(d-u)$, we obtain

$$
\begin{aligned}
1-\xi_{\text {unif }, d}(1-u) & =\sum_{i=-d}^{d-1} B^{d}((1-u) d-i)-\sum_{i=0}^{d-1} B^{d}((1-u) d-i) \\
& =\sum_{i=-d}^{-1} B^{d}(d u+i)=\sum_{i=0}^{d-1} B^{d}(d u-i)=\xi_{\text {unif }, d}(u)
\end{aligned}
$$

We compute the maximal magnitude of $d$-th derivative of $\xi_{\text {unif,d }}(u)$, to compare with the other blending functions. Using the equation for a B-spline derivative $\frac{d}{d u} B^{p}(u)=B^{p-1}(u)-B^{p-1}(u-1)$, we obtain the expression

$$
\left(\xi_{\text {unif }, d}\right)^{(d)}(u)=d^{d} \sum_{i=0}^{d-1} \sum_{j=0}^{d}\left(\begin{array}{l}
d \\
j
\end{array}\right)(-1)^{j} B^{0}(d u-i-j)
$$

As $B^{0}(u d-\ell)$ is zero outside the interval $[\ell / d,(\ell+1) / d$, it is sufficient to consider the sum over $0 \leq \ell=$ $i+j \leq n$ to determine the derivative values on $[0,1]$. Setting $i=\ell-j$, and rearranging the terms, we obtain

$$
\left(\xi_{\text {unif }, d}\right)^{(d)}(u)=d^{d} \sum_{\ell=0}^{d-1} B(d u-l) \sum_{j=0}^{\ell}(-1)^{j}\left(\begin{array}{l}
d \\
j
\end{array}\right)=d^{d} \sum_{\ell=0}^{d-1} B(d u-l)(-1)^{\ell}\left(\begin{array}{c}
d-1 \\
\ell
\end{array}\right)
$$

where we use the combinatorial equality $\sum_{j=0}^{\ell}(-1)^{j}\left(\begin{array}{c}d \\ j\end{array}\right)=(-1)^{\ell}\left(\begin{array}{c}d-1 \\ \ell\end{array}\right)$. As $B(d u-l)$ is 1 on $[l / d,(l+1) / d]$, the magnitude of the derivative on each such interval is constant, and equal to $\left(\begin{array}{c}d-1 \\ \ell\end{array}\right)$, which is maximal for $\lceil(d-1) / 2\rceil$.

The properties of $\xi_{\text {unif,d }}$ are summarized in the following proposition:

Proposition 1. The blending function $\xi_{u n i f, d}$ is $C^{d-1}$ continuous and satisfies the boundary conditions (22) and symmetry condition (23). Its d-th derivative is piecewise constant and its maximum is $\left(\begin{array}{c}d-1 \\ \lceil(d-1) / 2\rceil\end{array}\right)$. 


\begin{tabular}{|c|c|c|c|c|c|c|c|c|}
\hline $\mathrm{d}$ & 1 & 2 & 3 & 4 & 5 & 6 & 7 & 8 \\
\hline$r(h)$ & 1.95 & 2.74 & 5.34 & 13.2 & 39.4 & 179. & 904. & 4979 \\
\hline$r\left(\xi_{\text {unif,d }}\right)$ & 1.00 & 1.00 & 1.69 & 2.00 & 3.05 & 3.80 & 5.59 & 7.11 \\
\hline$r\left(\xi_{2, d}\right)$ & 1.00 & 1.50 & 1.88 & 2.19 & 2.46 & 2.71 & 2.93 & 3.14 \\
\hline $\sup _{[0,1]}\left|\xi_{\infty, d}\right|$ & 1 & 4 & 32 & 384 & 6144 & $1.22 \mathrm{e}+05$ & $2.94 \mathrm{e}+06$ & $8.25 \mathrm{e}+07$ \\
\hline
\end{tabular}

Table 1: Ratios $\left.r(\xi)=\sup _{[0,1]} \mid \xi^{(d)}\right)\left|/ \sup _{[0,1]}\right|\left(\xi_{\infty, d}\right)^{(d)} \mid$ for $h$, $\xi_{\text {unif,d }}$ and $\xi_{2, d}$; the last row shows values of $\sup _{[0,1]}\left|\left(\xi_{\infty, d}\right)^{(d)}\right|$.

$W_{2}^{d}$-optimal blending function. In this case we compute the function minimizing the functional

$$
\int_{0}^{1}\left(\xi^{(d)}(u)\right)^{2} d u
$$

with boundary conditions (22). The standard calculus of variations derivation yields the Euler-Lagrange equation for this functional:

$$
\xi^{(2 d)}=0
$$

We conclude that the solution of the optimization problem $\xi_{2, d}$ is a polynomial of degree $2 d-1.2 d$ boundary conditions define this polynomial uniquely, and an explicit expression for this polynomial can be obtained by observing that its derivative $g(t)=f^{\prime}(t)$ is a polynomial of degree $2 d-2$ satisfying $2 d-2$ homogeneous boundary conditions $g^{(i)}(0)=g^{(i)}(1)=0$. One can easily check that $t^{d-1}(1-t)^{d-1}$ satisfies the desired boundary conditions, and, therefore, all possible $g(t)$ are of the form $C t^{d-1}(1-t)^{d-1}$.

The function $\xi_{2, d}(t)$ can be obtained by integrating $g(t)$ over the interval $[0 \ldots t]$, and computing the constant $C$ using the condition $\xi_{2, d}(1)=1$. This leads to the formula

$$
\xi_{2, d}(u)=\frac{(2 d-1) !}{((d-1) !)^{2}} \sum_{i=0}^{d-1} \frac{(-1)^{i}}{i+d} u^{i+d}\left(\begin{array}{c}
d-1 \\
i
\end{array}\right)
$$

To compute the maximum of the derivative of $\xi_{2, d}$, we observe that it is attained at one of the boundary points 0 and 1 . While we do not prove this in the general case, this fact is easily verified numerically for any finite $d$ computing the roots of $\left(\xi_{2, d}\right)^{d+1}$ with guaranteed bounds and evaluating $\left(\xi_{2, d}\right)^{d}$. (We have verified this for $d \leq 20)$. The analytic expression for the value $\left|\left(\xi_{2, d}\right)^{(d)}\right|$ is easily obtained by differentiating (25).

Proposition 2. The blending function $\xi_{2, d}$ is $C^{d-1}$ continuous and satisfies the boundary conditions (22) and symmetry condition (23). Its d-th derivative is a polynomial of degree $d-1$, its maximum is $\frac{(2 d-1) !}{(d-1) !}$.

$W_{\infty}^{d}$-optimal blending function. As the magnitude of the pointwise values of the derivatives is often most important for applications, we consider blending functions defined as solutions of the following optimization problem:

$$
\sup _{u \in[0,1]}\left|\xi^{(d)}(u)\right| \rightarrow \min
$$

subject to $(22)$.

This type of blending function is a perfect spline. It properties are summarized in the following proposition:

Proposition 3 ( Glaeser, Louboutin (Glaeser, 1967), also found in Schoenberg (1971)). The blending function $\xi_{\infty, d}$, solving (22), is $C^{d-1}$-continuous and satisfies the boundary conditions (22) and symmetry condition (23). It is piecewise-polynomial of degree $d$, with points of discontinuity of d-th derivative are $t_{i}=\frac{1}{2}\left(1-\cos \frac{\pi}{d}\right)$. Its d-the derivative maximum is $2^{2 d-2}(d-1)$ !. 
Explicit expressions for the maximal derivative values in Propositions 1-3 allow us to compare different blending functions. The ratios of maximal derivative magnitudes to the $W_{\infty}^{2}$ for the range $d=1 \ldots 8$ are shown in the Table 1 . Note that while the ratio grows, for low values of $d$, the difference between polynomial blending functions is small; but all of them have significantly lower derivatives than $h$. We also observe a fundamental limitation of compactly supported functions: the magnitude of the derivatives above 6 of the optimal function can be regarded as infinite for most practical purposes.

Figure 12(a) compares different types of blending functions for $d=3$. Figure $12(\mathrm{~b})$-(f) shows the plots of $d$-th derivatives of all blending functions of smoothness $d-1$ (note that $W_{2}^{d}$-optimal function is a piecewise $C^{d-1}$ continuous polynomial of degree $2 d-1$ ).

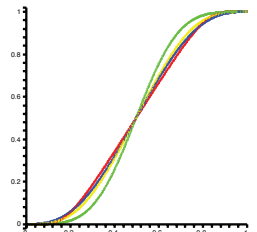

(a)

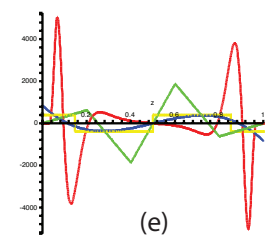

(e)

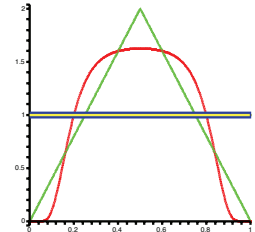

(b)

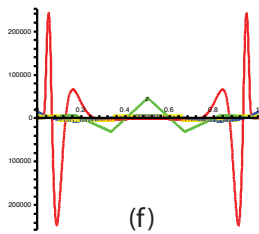

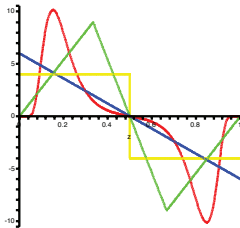

(c)

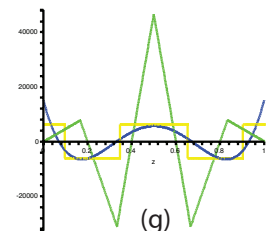

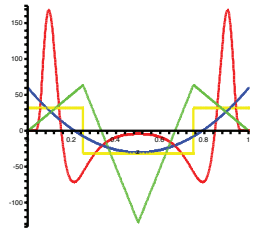

(d)

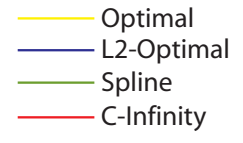

Figure 12: Comparison of different blending functions. (a) Function plots (b)-(f): Derivatives of order $d$ for $C^{\infty}$, uniform spline of degree $d, W_{2}^{d}$ - and $W_{\infty}^{d}$-optimal blending functions for $d=1 \ldots 5$. (g) Fifth derivative with the $C^{\infty}$ blending function omitted.

\section{Properties of manifold surfaces}

In the preceding sections we have discussed the possible choices for geometry and POU functions used in the manifold surface construction. We examine several properties of the resulting surfaces: numerical smoothness, flexibility and visual quality.

Our goal (cf. Section 1) is to construct surfaces of high-order smoothness, with explicit $C^{\infty}$ or $C^{d}$ parametrization, are at least 2-flexible, and are similar in visual quality to Catmull-Clark surfaces. We evaluate our construction according to these criteria.

\subsection{Parametrization Derivatives}

By construction, derivatives of all orders are formally defined for $C^{\infty}$ surface parametrization and derivatives up to order $d$ are defined for $C^{d}$ surfaces. However, from a practical point of view, large derivative magnitudes are indistinguishable from the absence of differentiability.

The tight lower bound on the maximal derivative norm for a compactly supported function $f$ with fixed $L_{2}$ norm, follows from the bounds on the derivative of the optimal blending function (3). Indeed, if $f$ is $C^{d-1}$ and supported on an interval $[0,1]$, we can introduce an auxiliary function $F(t)=\int_{0}^{t} f(s) d s$, the $L_{2}$ norm constraint becomes $F(1)=1$, and the problem reduces to the problem of finding the optimal blending function. This yields the upper bound for the derivative $2^{2 d} d$ !. This bound is relatively conservative, and is reached only for a bump of minimal support (e.g, with $z$ coordinate for one control point set to 1 and the rest to zero). To gain a better sense of the derivative behavior, we evaluate derivatives for different types of charts for a simple smooth shape.

The derivative magnitude range and distribution is shown in Figures 13, 14, 15 and 16. We consider derivatives up to order 5: as Table 1 indicates, derivatives of higher order are likely to have very large magnitudes. Figure 13 shows the behavior of derivatives on an interior chart of valance 6 for a $C^{\infty}$ surface(above) 
and $C^{5}$ surface(below). The remaining figures compares the behavior for smooth boundary, convex corner and concave corner charts. We observe that for $C^{5}$ spline-based construction we get a substantial improvement in derivative behavior primarily thanks to the change in the blending function.

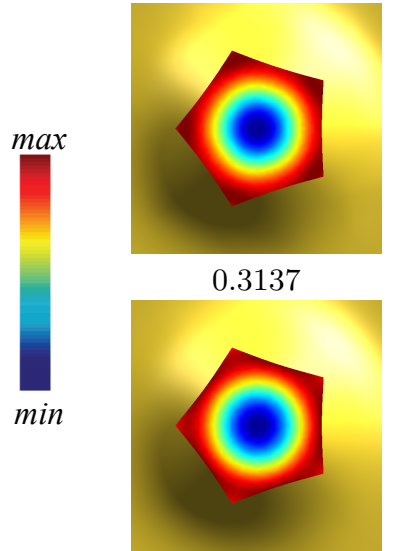

0.3136

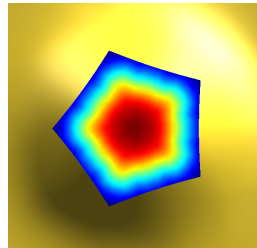

0.4648

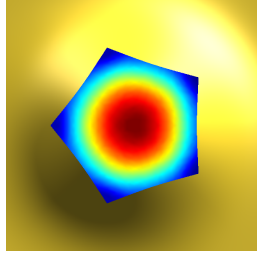

0.4500

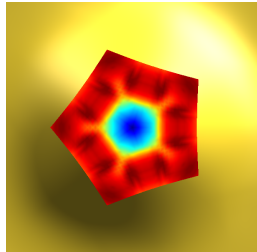

0.7030

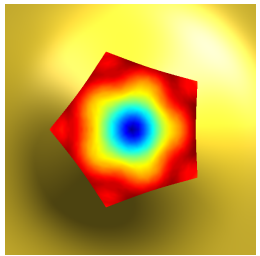

0.6793

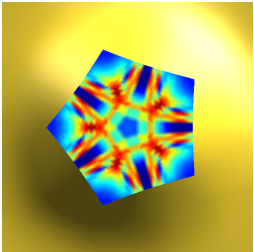

6.450

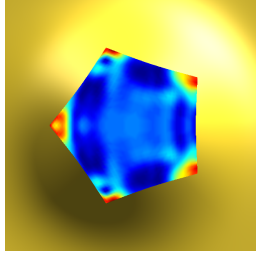

3.458

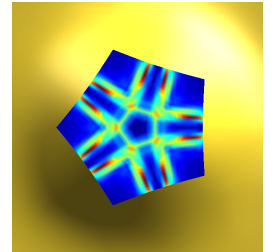

239.3

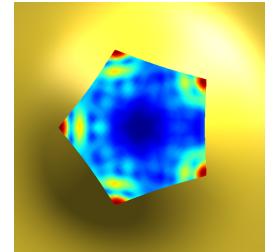

37.98

Figure 13: Distribution of derivative magnitudes for a $C^{\infty}$ (upper row) and a $C^{5}$ surface with $g=3$ (lower row); the maximal derivative magnitude is indicated below each image. The control mesh is flat with a single interior extraordinary vertex of valence 5 raised to height 1.

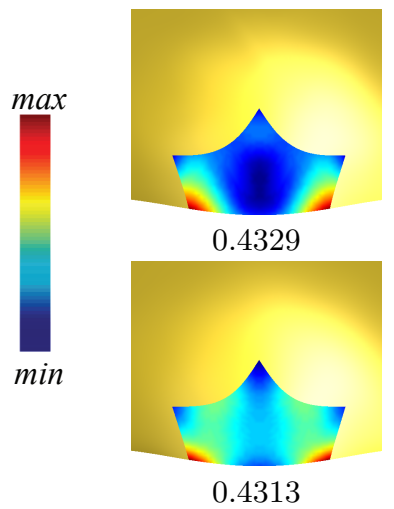

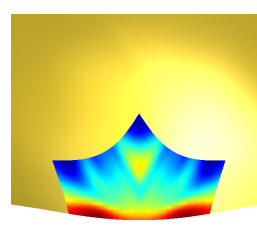

0.3343

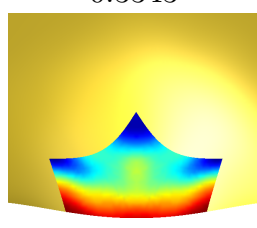

0.2833

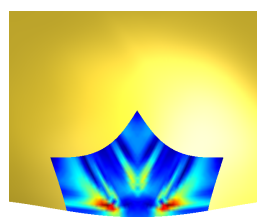

2.206

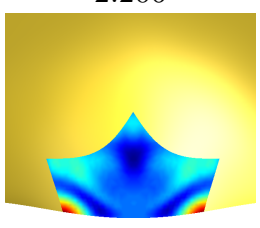

1.857

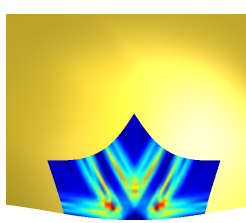

54.22

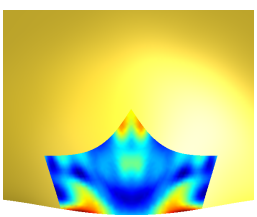

11.22

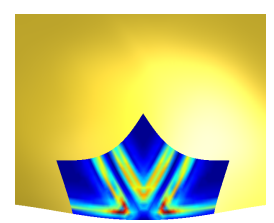

2142.

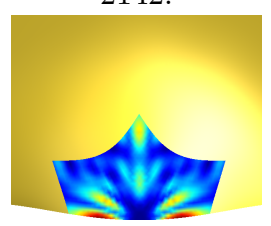

187.8

Figure 14: Distribution of derivative magnitudes for a $C^{\infty}$ (upper row) and a $C^{5}$ surface (lower row) with boundary $(g=3)$. The maximal derivative magnitude is indicated below each image. The control mesh is flat with a single smooth boundary extraordinary vertex of valence 3 raised to height 1 .

\subsection{Flexibility}

$C^{d}$-continuity only guarantees that the surface parametrization derivatives exist, but does not preclude undesirable behavior even locally: second or even first derivatives may vanish at certain fixed points of the surface for any choice of control point positions. This has been recognized in the context of subdivision surfaces (Reif, 1995): while it is relatively straightforward to construct $C^{2}$ subdivision surfaces with flat points at extraordinary vertices (Biermann et al., 2000b), (i.e., vanishing curvatures and second derivatives of a parametrization), it is far more difficult to define subdivision surfaces which have nonzero curvatures and 2nd derivatives of a suitable parametrization at the extraordinary vertices. The absence of 2-flexibility can be considered a major downside for a basis used to define surfaces, as it leads to visual artifacts and numerically undesirable behavior. 


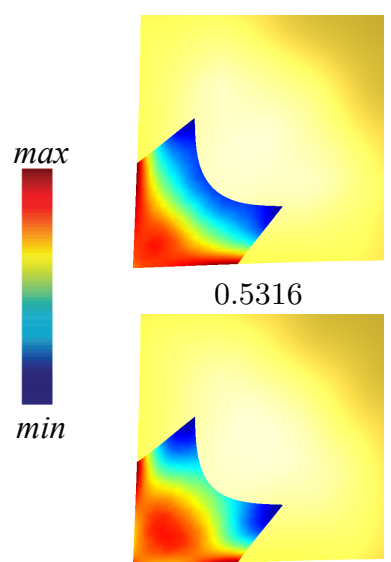

0.5196

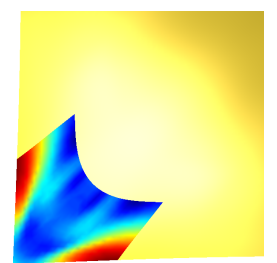

0.9555

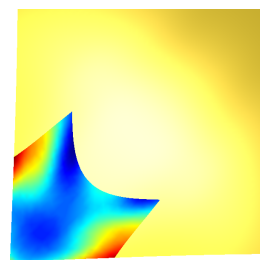

0.8212

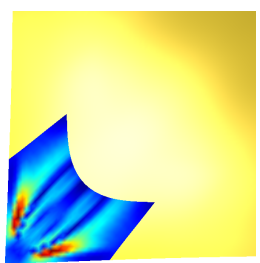

8.951

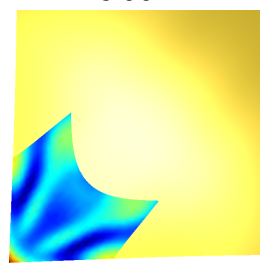

6.100

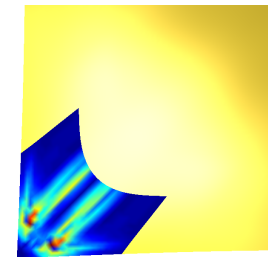

320.4

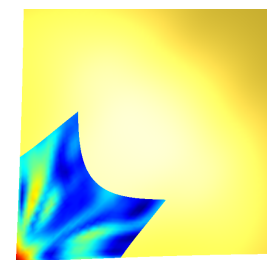

56.24

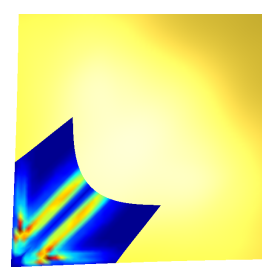

14800.

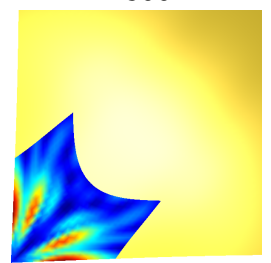

1289.

Figure 15: Distribution of derivative magnitudes for a $C^{\infty}$ (upper row) and a $C^{5}$ surface (lower row) with a convex corner $(g=3)$. The maximal derivative magnitude is indicated below each image. The control mesh is flat with a single convex corner extraordinary vertex of valence 3 raised to height 1 .

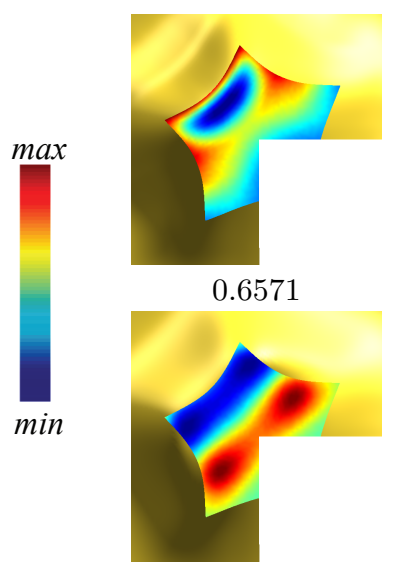

0.5583

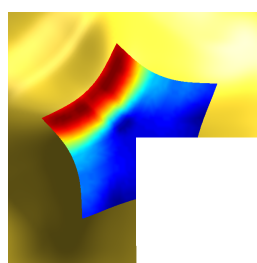

1.897

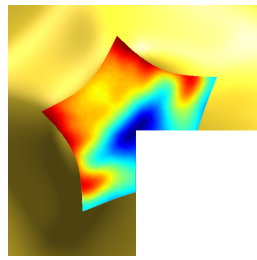

1.005

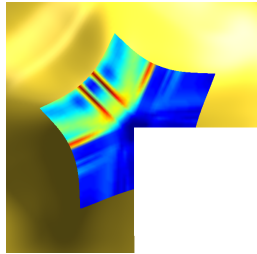

10.40

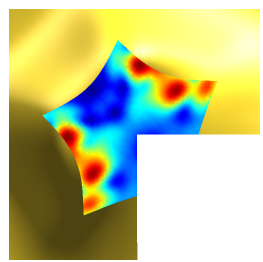

6.983

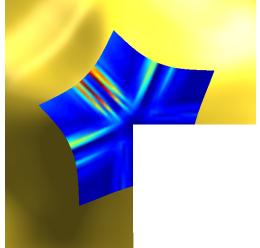

257.8

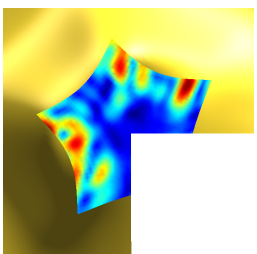

56.81

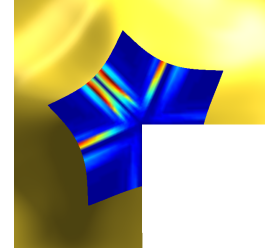

11150.

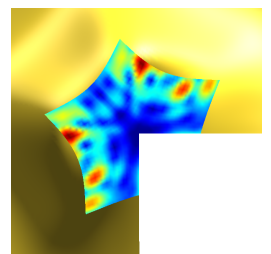

831.2

Figure 16: Distribution of derivative magnitudes for a $C^{\infty}$ (upper row) and a $C^{5}$ surface with $g=7$ (lower row) with a convex corner. The maximal derivative magnitude is indicated below each image. The control mesh is flat with a single convex corner extraordinary vertex of valence 3 raised to height 1 .

Definition 1. We say that a set of $C^{d}$-continuous functions $B_{i}(u, v),(u, v) \in \Omega \subset \mathbf{R}^{2}, i=1 \ldots n$, is $k$-flexible at a point $x=(u, v)$ if for any derivative $\partial_{u}^{\ell} \partial_{v}^{m}, l+m=k$, the span $\left\langle\sum_{i=0}^{n} c_{i} \partial_{u}^{l} \partial_{v}^{m} B_{i}(x), l+m \leq\right.$ $k\rangle=\mathbf{R}^{N}$, where $N=k(k+1) / 2$ is the number of derivatives of order $\leq k$. In other words, for all derivative of order $\leq k$ any desired set of values can be obtained simultaneously by a choice of control points.

In matrix form, $\sum_{i} c_{i} B_{i}(u, v)=B(u, v)^{T} c$. If $M[B]$ is the $N \times n$ matrix with rows equal to $\partial_{u}^{\ell} \partial_{v}^{m} B(u, v)$, then flexibility of the set $B_{i}$ is equivalent to the condition that matrix $M[B]$ has rank $N$.

One can easily show that manifold surfaces are at least 3-flexible by construction at the centers of charts, as the value of the surface there is defined by the local geometry function only (Ying and Zorin, 2004b). This does not guarantee however that the surface is flexible elsewhere. Flexibility at arbitrary points is in general difficult to prove analytically (although an analytic proof for a $C^{2}$ construction of Zorin (2006a) for meshes with nonadjacent extraordinary vertices is given in (Zorin, 2006b)).

For our construction, we examine flexibility at arbitrary points numerically. We focus on 2-flexibility, as it is most relevant in applications, although our technique can be used for flexibility of arbitrary order. Our numerical estimates cannot be regarded as a proof, but if desired could be converted to a formal proof of 
flexibility at a dense set of sample points by using interval arithmetic for all calculations, similar to (Zorin, 2000).

The local geometry function on a chart can be expressed as:

$$
f^{\text {Lock }}(x)=B^{T}(x) W p^{(c, 2)}=B^{T}(x) W S p^{c}
$$

where $p^{(2)}$ are the set of data points after two steps of subdivision, $p^{c}$ are the control points of the initial mesh $M$ in the 2-neighborhood of the vertex, which determine $p^{(c, 2)}$ uniquely.

Recall that the domain of our surface is the mesh $M$; on each face $F$ of $M$, we can introduce bilinear coordinates $x=(u, v)$, in the range $[0,1]$. For a surface patch corresponding to a face $F$, the direct formula for the surface $f$ is obtained by blending local geometry functions (similar to (1), but with face, rather than chart, coordinates considered):

$$
f=\sum_{i=1}^{4}\left(f^{l o c} w_{i}\right) \circ \varphi_{i}^{-1}=\sum_{i=1}^{4}\left(\left(B^{i}\right)^{T} w_{i}\right) \circ \varphi_{i}^{-1} W^{i} S^{i} p^{c, i}
$$

where the subscript $i$ refer to the chart centered at vertex $i=1 \ldots 4$ of the face $F$. Let $p$ be the vector of all points in 2-neighborhood of $F$. Each vector $p^{c, i}$ can be obtained by selecting entries from $p$, so we can write $p^{c, i}=Q_{i} p$, where $Q_{i}$ is the selecting matrix with 0 and 1 entries. This leads to a combined expression for $f(x)$ on $F$ :

$$
f=\left(\sum_{i=1}^{4}\left(w_{i}\left(B^{i}\right)^{T}\right) \circ \varphi_{i}^{-1} W^{i} S^{i} Q^{i}\right) p=\left(B^{F}\right)^{T} p
$$

where $B^{F}$ is the vector of basis functions corresponding to vertices in the 2-neighborhood of the face $F$ in $M$. These functions depend only on vertex valences and chart types overlapping $F$, but not on control point positions. Constructing the matrix $6 \times n M\left[B^{F}\right]$ with 6 rows $\partial_{u}^{\ell} \partial_{v}^{m} B^{F}, \ell+m \leq 2$, we reduce the question of verifying 2-flexibility to verifying that this matrix has maximal rank for any point on $F$ and any choice of valences and chart types.

While it is possible to estimate the rank of matrices $M\left[B^{F}\right]$ numerically using SVD, we simplify our task by applying a basis transformation to the basis $\tilde{B}$ with the new basis chosen using geometric considerations. To motivate our choice, we consider a simple example of polynomial basis $P 1, u, v, u^{2}, u v, v^{2} \ldots$, up to polynomials of total order $k$. Let $M[P]^{6}$ be the $6 \times 6$ matrix obtained from the full $6 \times n$ matrix $M[P]$ by taking the first 6 columns. One can easily see that it is an upper-triangular matrix with constant non-zero entries on the diagonal (reducing to a diagonal matrix for $(u, v)=(0,0)$ ), and, therefore, has rank 6 . We conclude that $M[P]$ also has rank 6 .

While $B^{F}$ does not reproduce polynomials other than the constant, it is reasonable to expect that a configuration of control points $c_{i}$ sampled from a polynomial will lead to a function $\sum_{i} c_{i} B_{i}^{F}$ with qualitative behavior similar to this polynomial. If we replace $B^{F}$ with a set of functions $\tilde{B}$ approximating polynomials, one can hope to establish flexibility in most cases by examining only the determinant of a $6 \times 6$ submatrix $M[\tilde{B}]^{6}$ of the full matrix of derivatives $M[\tilde{B}]$ and consider other submatrices only rarely. In this way, we can check flexibility at any given point by evaluating a small number (typically one) matrix determinants.

To test flexibility over the whole face $F$, we evaluate the determinant $M[\tilde{B}]^{6}$ on a dense grid, and verify that all signs are the same. If the determinant changes sign, we replace one row with a different row from $M[\tilde{B}]$ and repeat the process for the new matrix.

Transformed basis construction. For a 2-neighborhood of face $F$, we construct the transformed basis functions $\tilde{B}_{i}=\sum_{j} c_{i j} B_{i}$ in two steps. First, we construct the one-to-one map of the 2-neighborhood of $F$ to the plane, with positions of points entirely determined by the mesh connectivity, and $F$ mapped to the square $[0,1]^{2}$ (we discuss this step in more detail below). Each vertex gets coordinates $u_{i}$ and $v_{i}$; we use these coordinates to construct our approximations of linear polynomials $\tilde{B}_{u}=\sum_{j} u_{j} B_{j}$ and $\tilde{B}_{v}=\sum_{j} v_{j} B_{j}$. Approximations of higher-order polynomials $P^{\ell, m}(u, v)=u^{m} v^{l}$ are constructed as $\tilde{B}_{u^{m}} v^{l}=\sum_{j} v_{j}^{m} u_{j}^{l} B_{j}$, that is, by using sampled values $P^{\ell, m}\left(u_{i}, v_{i}\right)$ at vertices of the 2-neighborhood of $F$. The basis transformation can be expressed in matrix form as $\tilde{B}(u, v)=T B(u, v)$ where $T$ is the matrix with entries $v_{j}^{m} u_{j}^{l}$. 
It remains to describe how the positions $\left(u_{i}, v_{i}\right)$ are determined. We restrict our construction to the case of meshes obtained by 1 step of Catmull-Clark refinement from an arbitrary mesh, to separate extraordinary vertices by vertices of valence 4 , as otherwise the number of possible distinct connectivities of 2-neighborhood of a patch is very high (if desired, e.g., the Floater parametrization method can be used to determine $u_{i}$ and $v_{i}$ for an arbitrary mesh). For our restricted setting, a simple construction shown in Figure 17 suffices. Let $G$ be the parent patch of $F$, and let $v$ be the vertex of $G$ shared with $F$. We map the 1-neighborhood of $G$ to the plane, by mapping the 1-neighborhood of $v$ to a symmetric star first, and then mapping the remaining quads adjacent to $G$ so that they are mapped to parallelograms of equal size. Finally, a single step of midpoint subdivision is used to obtain the planar layout.
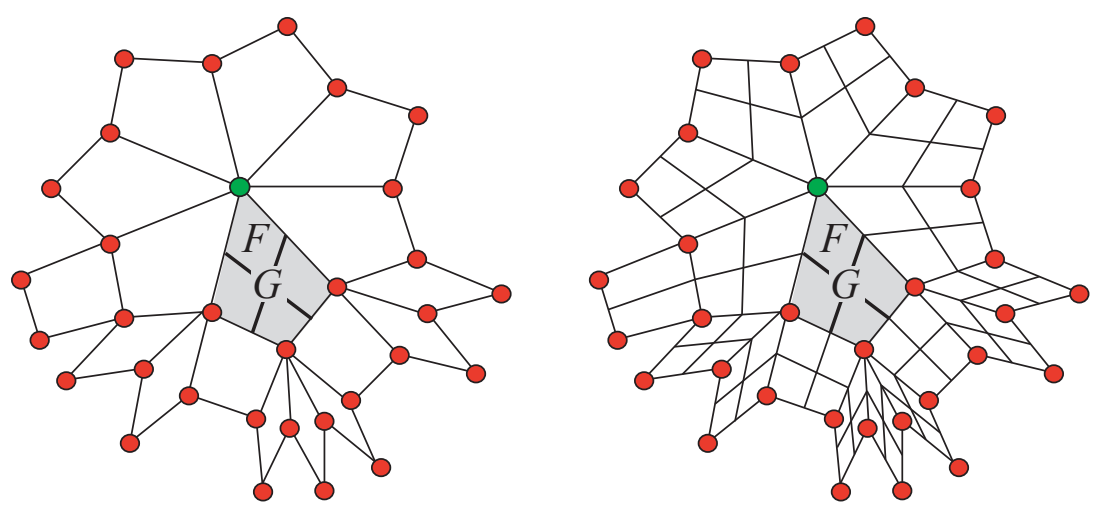

Figure 17: Construction of the planar map of a 2-neighborhood of a face for valences $\{7,5,6,5\}$ in a mesh with no adjacent extraordinary vertices. Left: initial layout for 1-neighborhood of the parent $G$ of $F$. Right: layout for the 2-neighborhood of $F$.

Numerical verification of 2-flexibility. If only one vertex in the 2-neighborhood of $F$ is extraordinary, the minimal $\operatorname{det} M^{6}[\tilde{B}]$ as a function of the valence is shown in Figure 18, right). Figure 18 shows the value of the determinant as function of the coordinates $(u, v)$ on the face $F$.
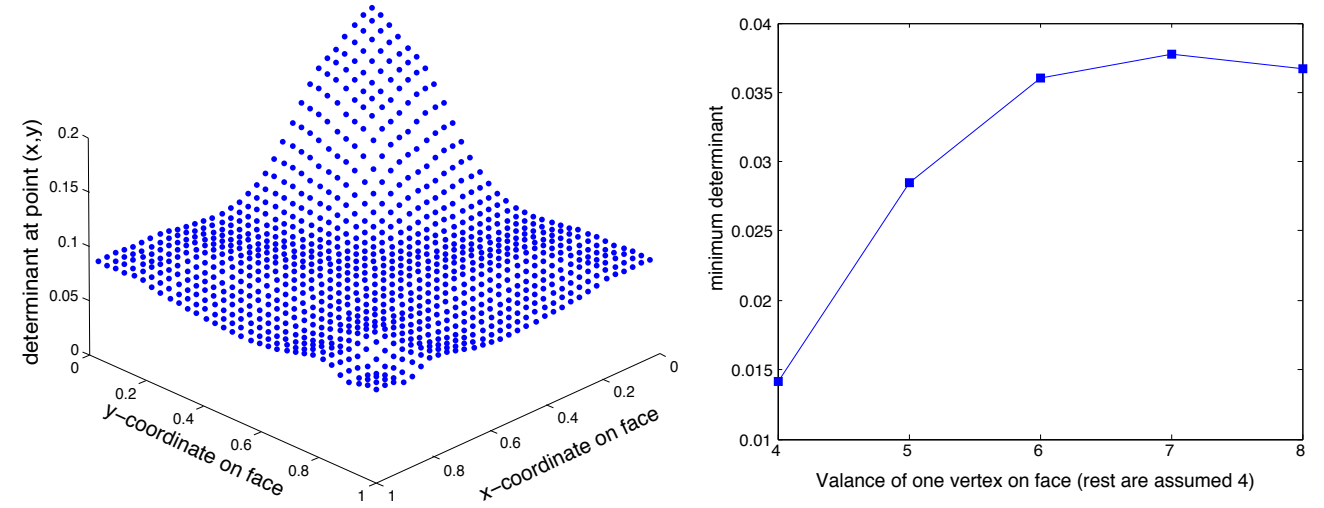

Figure 18: Left: Determinant distribution on a face $f$ with one valence six vertex at $(0,0)$. Right: Plot of minimum determinant on a face as a function of valance.

We have tested all combinations of valances for the four vertices surrounding the face $F$ for valences in the range 3..12, in all cases observing that the determinant $\operatorname{det} M^{6}[\tilde{B}]$ is nonnegative. For valances higher than $13 \operatorname{det} M^{6}[\tilde{B}]$ may change sign, and other submatrices using a different set of six columns. In all cases we could successfully find other submatrices where the positivity of the determinants are maintained. 


\subsection{Visual quality}

$C^{d}$-continuity and flexibility are local surface properties and do not capture the global surface behavior. It is well-known that a formally $C^{2}$ or higher-order surface construction can exhibit severe artifacts that preclude its use in practical applications. While various types of formal measures of global behavior can be developed, visual comparison is often a more reliable criterion.

By using sample points from a Catmull-Clark subdivision surface, we ensure that the overall shape of the manifold surface is close to the shape of the reference subdivision surface. However, relatively small deviations may lead to subtle changes in surface appearance. The differences typically cannot be seen even with specular shading, and we use reflection lines to show these clearly.

Figure 19 compares the appearance of Catmull-Clark $C^{\infty}, C^{k}$-continuous surfaces build using our construction. Only insignificant differences in reflection line patterns can be found in most cases. For some models, one can identify areas where the reflection lines for higher-order $C^{k}$ surfaces are less even (Figure 20).

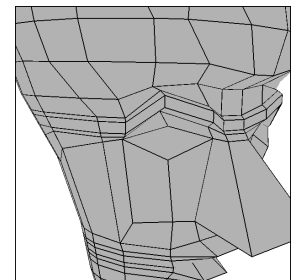

Control mesh

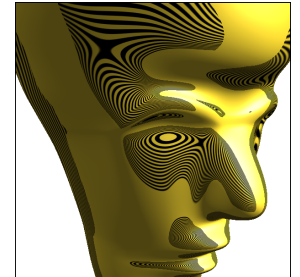

Catmull-Clark

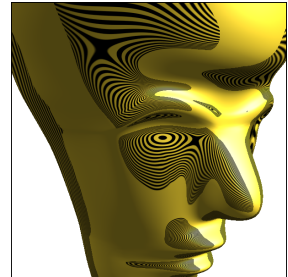

$C^{\infty}$

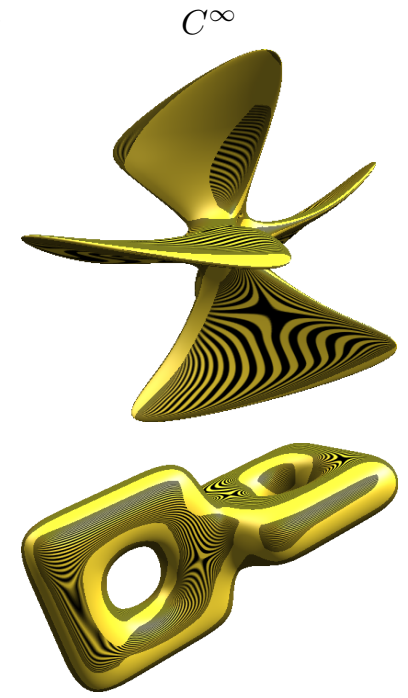

Catmull-Clark

$C^{k}$

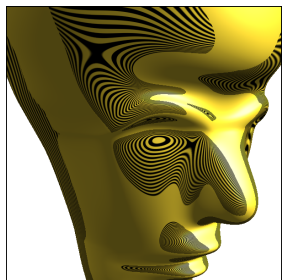

$C^{3}$

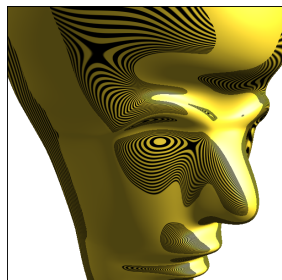

$C^{5}$

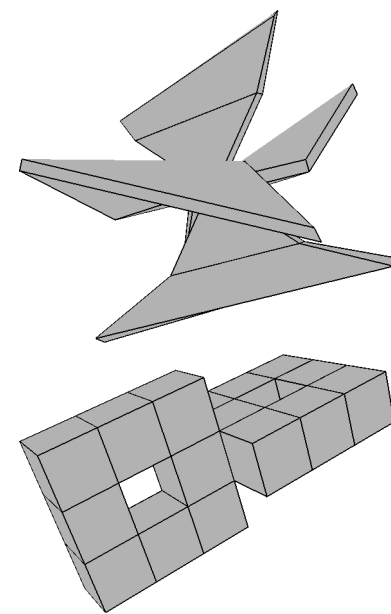

Control mesh

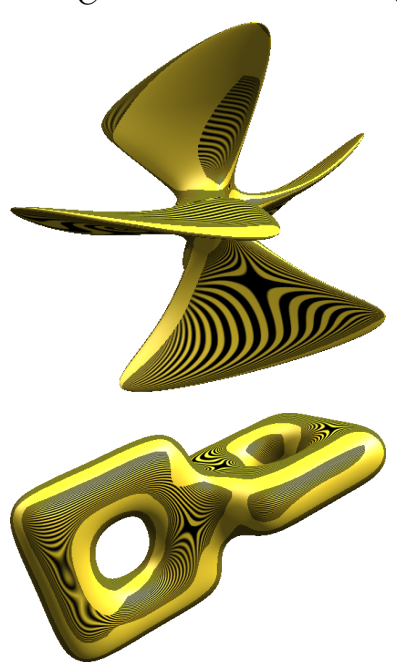

$C^{5}$

Figure 19: For most meshes, overall Catmull-Clark surface and $C^{k}$ surface appearance are nearly identical, with some differences apparent in flat areas (e.g., for the double torus).

The two main parameters of our spline construction are the degree of the spline and the number of spline patches we use; In most cases (with an important exception of concave corners) the number of patches makes little difference, as long as it is not a single patch, and the visual appearance of surfaces for $k$ in the range 2 to 5 is similar (Figure 21).

Figure 23 compares the behavior of the reflection lines for (unmodified) Catmull-Clark $C^{3}$, and $C^{\infty}$ surfaces near boundary vertices of valence 3,5 and 7 .

Figure 22 compares the overall appearance of Catmull-Clark, $C^{\infty}$ manifold surfaces and $C^{3}$ manifold surfaces for concave corners. One can once again see the limitations of the $C^{\infty}$ approach for concave corners: even relatively high polynomial degree is insufficient to match the overall shape of the subdivision surface closely, resulting in an overshoot. With sufficiently many patches, the spline surface results in a closer match, although the deviation from the subdivision surface is still significant. 


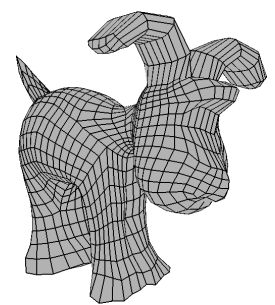

Control mesh

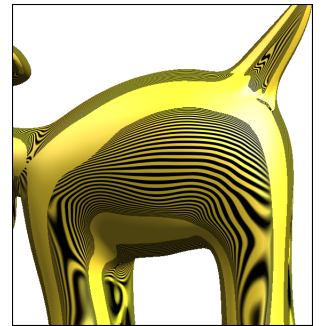

Catmull-Clark

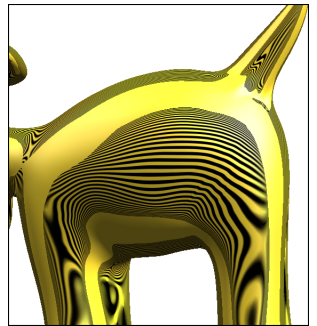

$C^{\infty}$

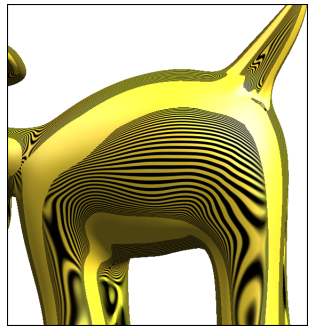

$C^{3}$

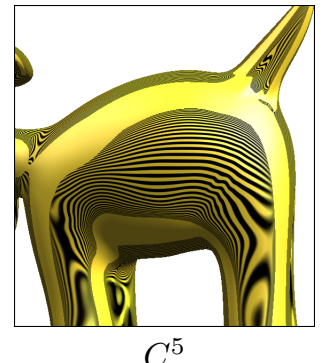

$C^{5}$

Figure 20: On some meshes $C^{\infty}$ and higher-order (e.g., $C^{5}$ ) surfaces have minor artifacts in relatively flat areas absent in lower-order $\left(C^{2}\right.$ or $\left.C^{3}\right)$ manifold surfaces and Catmull-Clark surfaces.

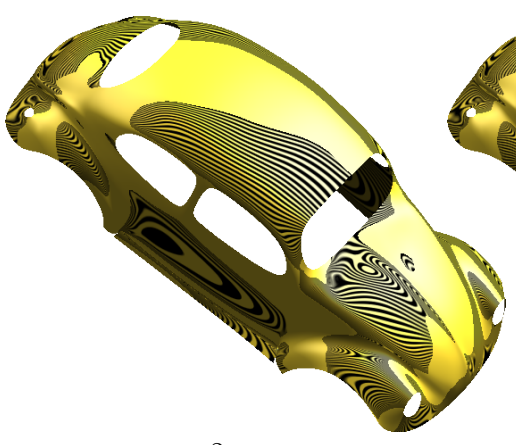

$C^{2}, g=3$

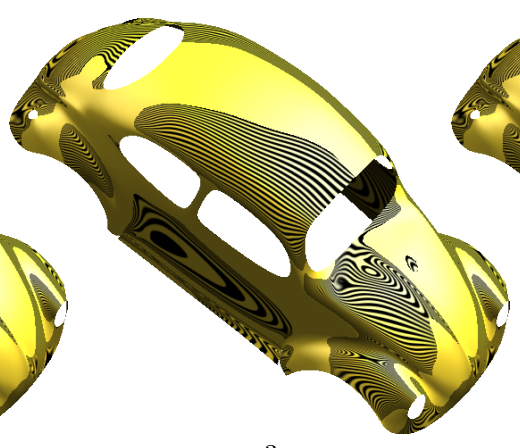

$C^{3}, g=3$

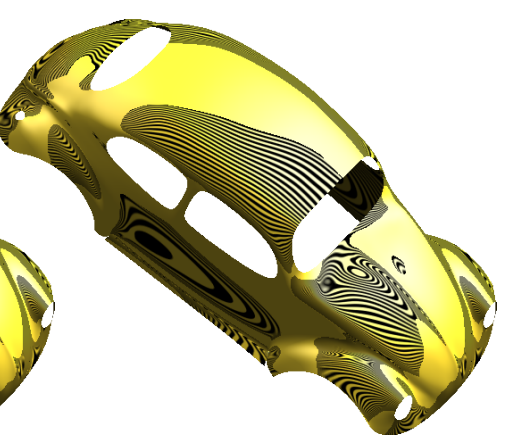

$C^{5}, g=3$

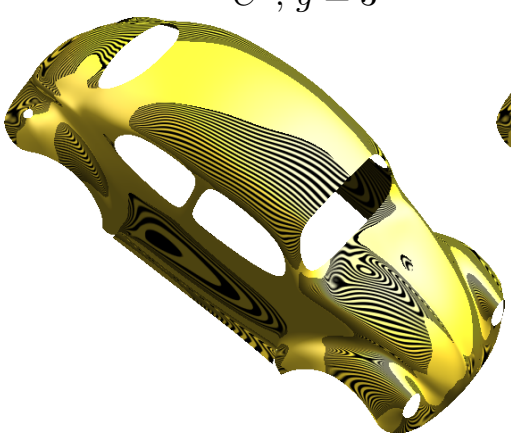

$C^{3}, g=3$

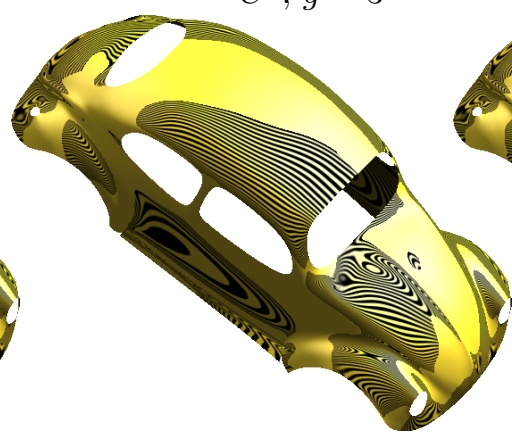

$C^{3}, g=5$

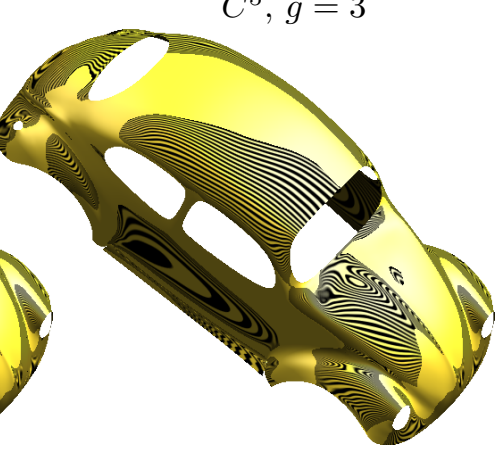

$C^{3}, g=7$

Figure 21: Upper row: surface appearance for fixed $g=3$ and varying smoothness; Lower row: fixed smoothness $C^{3}$ and varying $g$

Figure 24 compares the effects of changing face aspect ratio on Catmull-Clark and $C^{3}$ surfaces. In the top row, the mesh contains more elongated faces; in this case the reflection lines for the manifold surface are noticeably less fair compared to the Catmull-Clark surface. For a mesh with more uniform face aspect ratios, the difference is small.

\section{Conclusion}

We presented a manifold-based smooth surface construction which can handle all basic types of piecewise smooth boundary behavior. Similarly to splines, it can be evaluated using a closed form parametrization (although a significantly more complex one) allows choosing the degree of smoothness of the parametrization, and exhibits nearly-optimal rate of growth of high-order derivatives for the class of parametric surfaces defined on meshes with basis function support restricted to two rings around vertices. While it is difficult 


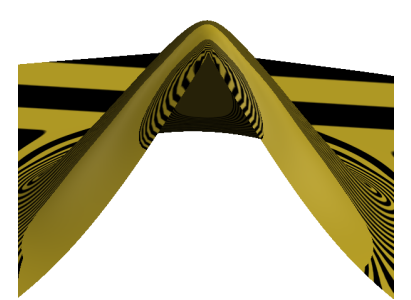

Catmull-Clark

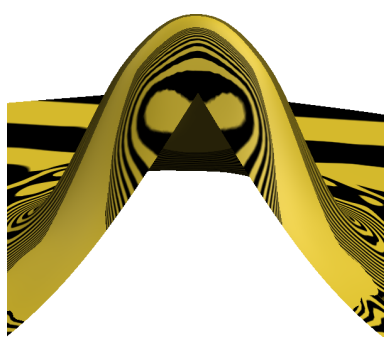

$C^{\infty}$

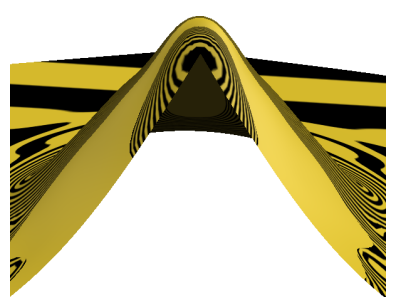

$C^{3}, g=11$

Figure 22: A close-up of a concave corner of valence 3 (flat mesh with the corner vertex lifted to height 1) for different surface definitions.

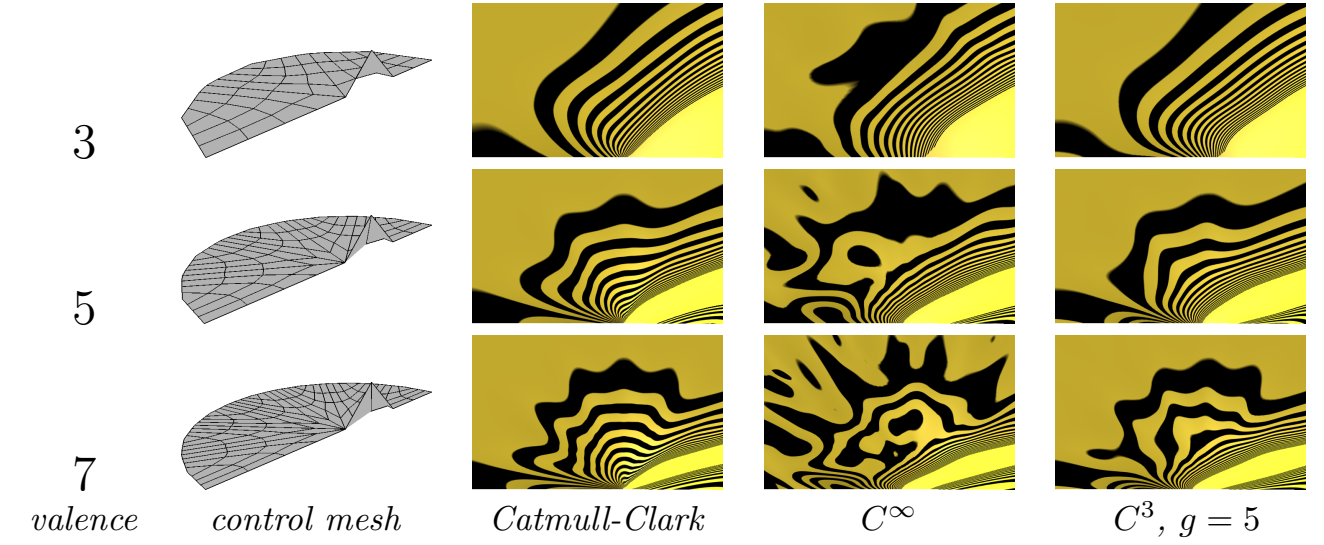

Figure 23: Comparison of surface behavior for smooth boundary vertices of valences 3,5 and 7 .
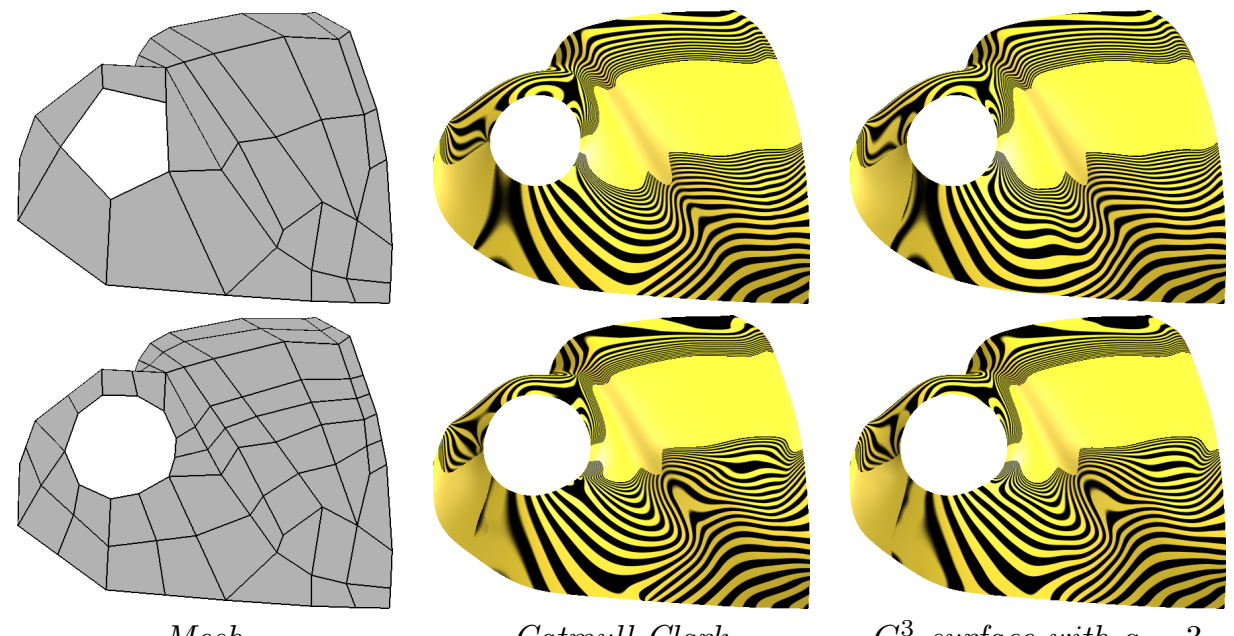

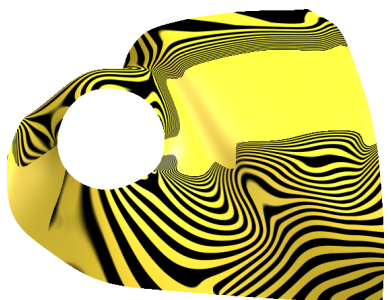

Catmull-Clark

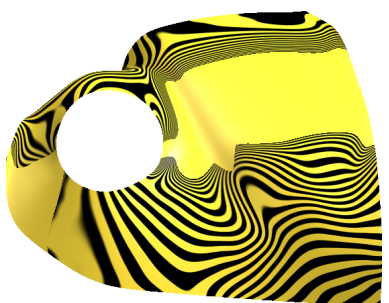

$C^{3}$ surface with $g=3$

Figure 24: High aspect-ratio faces may result in more irregular reflection lines on manifold surfaces compared to Catmull-Clark (upper row). Splitting such faces results in higher consistency with the appearance of the corresponding Catmull-Clark surface (lower row).

to prove flexibility of our surface construction at arbitrary points analytically, an extensive numerical study confirms that these surfaces are 2-flexible.

It appears unlikely that it is possible to improve the derivative behavior due to fundamental lower bounds 
on the derivative growth, but it may be possible to achieve this by increasing basis function support and chart size - a possible direction for future research. A fundamental limitation of our construction is using CatmullClark surfaces as the "guide" surface. This means that our surfaces inherit all artifacts of Catmull-Clark surfaces, e.g., severe problems near high-valence vertices. On the other hand, one can use our construction in combination with any coarse-mesh construction to obtain surfaces of arbitrary smoothness, so a more expensive or advanced technique yielding better quality surfaces can be used instead of Catmull-Clark.

We are able to match the visual quality and shape of Catmull-Clark surfaces very closely, with the exception of concave corners. It may be possible to obtain better results using a different choice of spline basis instead of tensor-product B-splines.

\section{References}

Biermann, H., Levin, A., Zorin, D., 2000a. Piecewise smooth subdivision surfaces with normal control. In: Akeley, K. (Ed.), Siggraph 2000, Computer Graphics Proceedings. ACM Press / ACM SIGGRAPH / Addison Wesley Longman, pp. 113-120.

Biermann, H., Levin, A., Zorin, D., 2000b. Piecewise smooth subdivision surfaces with normal control. In: SIGGRAPH 2000 Conference Proceedings. pp. 113-120.

Bohl, H., Reif, U., 1997. Degenerate bézier patches with continuous curvature. Comput. Aided Geom. Des. 14 (8), $749-761$.

Bruno, O. P., Kunyansky, L. A., 2001. A fast, high-order algorithm for the solution of surface scattering problems: basic implementation, tests, and applications. J. Comput. Phys. 169 (1), 80-110.

Glaeser, G. (Ed.), 1967. Le Prolongateur de Whitney, vol. I (1966), vol. II (1967). Université de Rennes.

Gregory, J. A., Hahn, J. M., 1989. A $C^{2}$ polygonal surface patch. Comput. Aided Geom. Design 6 (1), 69-75.

Grimm, C. M., 2002. Simple manifolds for surface modeling and parameterization. In: Shape Modeling International.

Grimm, C. M., Hughes, J. F., Aug. 1995. Modeling surfaces of arbitrary topology using manifolds. In: Proceedings of SIGGRAPH 95. Computer Graphics Proceedings, Annual Conference Series. pp. 359-368.

Grimm, C. M., Hughes, J. F., 2003. Parameterizating n-holed tori. In: The Mathematics of Surfaces IX. pp. 14-29.

Gu, X., He, Y., Jin, M., Luo, F., Qin, H., Yau, S.-T., 2007. Manifold splines with single extraordinary point. In: SPM '07: Proceedings of the 2007 ACM symposium on Solid and physical modeling. ACM, New York, NY, USA, pp. 61-72.

Gu, X., He, Y., Qin, H., 2005. Manifold splines. In: SPM '05: Proceedings of the 2005 ACM symposium on Solid and physical modeling. ACM Press, New York, NY, USA, pp. 27-38.

Gu, X., He, Y., Qin, H., 2006. Manifold splines. Graphical Models 68 (3), 237-254.

Hagen, H., Pottmann, H., 1989. Curvature continuous triangular interpolants. In: Mathematical methods in computer aided geometric design (Oslo, 1988). Academic Press, Boston, MA, pp. 373-384.

Karčiauskas, K., Peters, J., 2005. Polynomial $C^{2}$ spline surfaces guided by rational multisided patches. In: Computational methods for algebraic spline surfaces. Springer, Berlin, pp. 119-134.

Levin, A., 2006. Modified subdivision surfaces with continuous curvature. In: International Conference on Computer Graphics and Interactive Techniques. ACM Press New York, NY, USA, pp. 1035-1040.

Loop, C., 2004. Second order smoothness over extraordinary vertices. In: SGP '04: Proceedings of the 2004 Eurographics/ACM SIGGRAPH symposium on Geometry processing. ACM Press, New York, NY, USA, pp. 165-174.

Navau, J. C., Garcia, N. P., 2000. Modeling surfaces from meshes of arbitrary topology. Computer Aided Geometric Design 17 (7), 643-671.

Navau, J. C., Garcia, N. P., Anglada, M. V., 2002. A generic approach to free form surface generation. J. Comput. Inf. Sci. Eng. 2 (4), 294-301.

Peters, J., Feb 1996. Curvature continuous spline surfaces over irregular meshes. Computer-Aided Geometric Design 13 (2), $101-131$.

Peters, J., 2002. $C^{2}$ free-form surfaces of degree (3,5). Comput. Aided Geom. Design 19 (2), $113-126$.

Prautzsch, H., 1997. Freeform splines. Comput. Aided Geom. Design 14 (3), 201-206.

Prautzsch, H., Umlauf, G., 1998. A $G^{2}$-subdivision algorithm. In: Farin, G., Bieri, H., Brunnet, G., DeRose, T. (Eds.), Geometric Modelling, Computing Suppl. 13. Springer-Verlag, pp. 217-224.

Reif, U., 1995. A degree estimate for polynomial subdivision surface of higher regularity. Tech. rep., Universität Stuttgart, Mathematisches Institut A, preprint.

Schoenberg, I., 1971. The perfect B-splines and a time-optimal control problem. Israel Journal of Mathematics 10 (3), $261-274$.

Wang, H., Jin, M., He, Y., Gu, X., Qin, H., 2008. User-controllable polycube map for manifold spline construction. In: Proceedings of the 2008 ACM symposium on Solid and physical modeling. ACM New York, NY, USA, pp. 397-404.

Ying, L., Biros, G., Zorin, D., 2006. A high-order 3D boundary integral equation solver for elliptic PDEs in smooth domains. Journal of Computational Physics 219 (1), 247-275.

Ying, L., Zorin, D., 2004a. A simple manifold-based construction of surfaces of arbitrary smoothness. ACM Trans. Graph. $23(3), 271-275$.

Ying, L., Zorin, D., 2004b. A simple manifold-based construction of surfaces of arbitrary smoothness. ACM Trans. Graph. $23(3), 271-275$.

Zorin, D., 2000. A method for analysis of $C^{1}$-continuity of subdivision surfaces 37 (5), 1677-1708, sIAM Jornal of Numerical Analysis.

Zorin, D., 2006a. Constructing curvature-continuous surfaces by blending. Proceedings of the fourth Eurographics symposium on Geometry processing, 31-40.

Zorin, D., 2006b. Flexibility of curvature-continuous surfaces obtained by blending loop subdivision surfaces with quadratic patches. Tech. rep., New York University. 